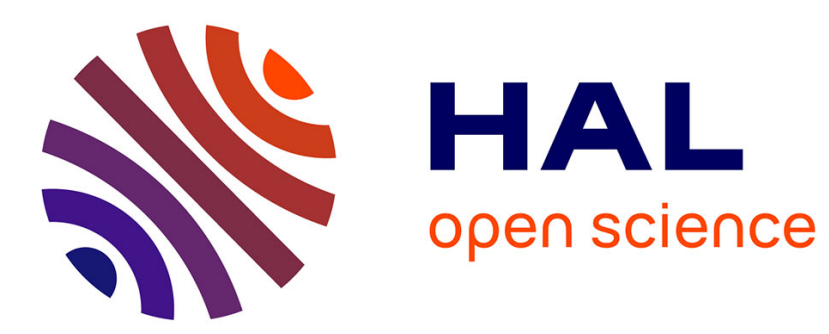

\title{
Complex magnetic order on the atomic scale revealed by spin-polarized scanning tunneling microscopy
}

Kirsten von Bergmann, Matthias Bode, Andre Kubetzka, Oswald Pietzsch, Elena Vedmedenko, Roland Wiesendanger

\section{- To cite this version:}

Kirsten von Bergmann, Matthias Bode, Andre Kubetzka, Oswald Pietzsch, Elena Vedmedenko, et al. Complex magnetic order on the atomic scale revealed by spin-polarized scanning tunneling microscopy. Philosophical Magazine, 2008, 88 (18-20), pp.2627-2642. 10.1080/14786430802337097. hal-00513941

\section{HAL Id: hal-00513941 \\ https://hal.science/hal-00513941}

Submitted on 1 Sep 2010

HAL is a multi-disciplinary open access archive for the deposit and dissemination of scientific research documents, whether they are published or not. The documents may come from teaching and research institutions in France or abroad, or from public or private research centers.
L'archive ouverte pluridisciplinaire HAL, est destinée au dépôt et à la diffusion de documents scientifiques de niveau recherche, publiés ou non, émanant des établissements d'enseignement et de recherche français ou étrangers, des laboratoires publics ou privés. 


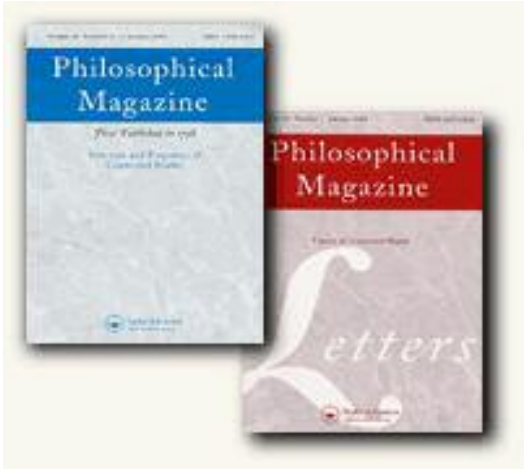

\section{Complex magnetic order on the atomic scale revealed by spin-polarized scanning tunneling microscopy}

\begin{tabular}{|c|c|}
\hline Journal: & Philosophical Magazine \& Philosophical Magazine Letters \\
\hline Manuscript ID: & TPHM-08-May-0171.R1 \\
\hline Journal Selection: & Philosophical Magazine \\
\hline $\begin{array}{l}\text { Date Submitted by the } \\
\text { Author: }\end{array}$ & 03-Jul-2008 \\
\hline Complete List of Authors: & $\begin{array}{l}\text { von Bergmann, Kirsten; University of Hamburg, Institute for } \\
\text { Applied Physics; University of Hamburg, Institute for Applied } \\
\text { Physics } \\
\text { Bode, Matthias; Argonne National Laboratory } \\
\text { Kubetzka, Andre; University of Hamburg, Institute of Applied } \\
\text { Physics } \\
\text { Pietzsch, Oswald; University of Hamburg, Institute for Applied } \\
\text { Physics } \\
\text { Vedmedenko, Elena; University of Hamburg, Institute for Applied } \\
\text { Physics } \\
\text { Wiesendanger, Roland; University of Hamburg, Institute of Applied } \\
\text { Physics }\end{array}$ \\
\hline Keywords: & magnetic order, nanostructures \\
\hline \multicolumn{2}{|l|}{ Keywords (user supplied): } \\
\hline \multicolumn{2}{|c|}{$\begin{array}{l}\text { Note: The following files were submitted by the author for peer review, but cannot be converted } \\
\text { to PDF. You must view these files (e.g. movies) online. }\end{array}$} \\
\hline Paper_Phil_Mag.tex & \\
\hline
\end{tabular}




\section{S. ScholarONE"}


Philosophical Magazine

Vol. 00, No. 00, 15 May 2008, 1-15

\title{
RESEARCH ARTICLE
}

\section{Complex magnetic order on the atomic scale revealed by spin-polarized scanning tunneling microscopy}

\author{
K. von Bergmann ${ }^{\mathrm{a}}$, M. Bode ${ }^{\mathrm{b}}$, A. Kubetzka ${ }^{\mathrm{a}}$, O. Pietzsch ${ }^{\mathrm{a}}$, E. Y. Vedmedenko ${ }^{\mathrm{a}}$ and R. \\ Wiesendanger ${ }^{\mathrm{a} *}$ \\ ${ }^{a}$ Institut for Applied Physics, University of Hamburg, Jungiusstr. 11, D-20355 Hamburg, \\ Germany; ${ }^{\mathrm{b}}$ Argonne National Laboratory Center for Nanoscale Materials $9700 \mathrm{~S}$ Cass \\ Ave Argonne, IL 60439-4806, USA \\ ( 44.1 released May 2008)
}

\begin{abstract}
A fundamental understanding of magnetic phenomena requires the determination of spin structures down to the atomic scale. The direct visualization of atomic-scale spin structures has been accomplished by combining the atomic resolution capability of Scanning Tunnelling Microscopy (STM) with spin sensitivity, based on vacuum tunnelling of spin-polarized electrons. The resulting technique, Spin-Polarized Scanning Tunnelling Microscopy (SP-STM), nowadays provides unprecedented insight into collinear and non-collinear spin structures at surfaces of magnetic nanostructures and has already led to the discovery of new types of magnetic order at the nanometer scale. Several examples of complex magnetic order as revealed by atomic-resolution SP-STM will be reviewed.
\end{abstract}

Keywords: Spin-Polarized Scanning Tunnelling Microscopy (SP-STM), vacuum tunnelling

\section{Introduction}

The investigation of magnetism in thin films and nanostructures on surfaces by spin-polarized scanning tunneling microscopy (SP-STM) has demonstrated the capability of this method to study ferromagnetic, ferrimagnetic or even antiferromagnetic structures down to the atomic scale [1-3]. Already the investigation of homoatomic monolayers of $3 d$ transition metals on $5 d$ substrates has revealed fascinating physics $[3,4]$ and allows an interpretation of the experimental results based on the atomic scale magnetic structure [5]. While the formation of a ferromagnetic or antiferromagnetic state can be the result of a dominating nearest neighbor exchange interaction, complex magnetic structures often reflect a situation of competing magnetic interactions. Exchange interactions in a homoatomic monolayer on a substrate can be described by mapping the results of ab-initio electronic structure calculations on a two-dimensional classical Heisenberg model. This makes it possible to search for the origin of a specific magnetic ground state, i.e. to find out which interactions dominate or compete. This of course facilitates a general understanding of magnetism and might allow the tailoring of magnetic properties in the future.

*Corresponding author. Email: rwiesend@physnet.uni-hamburg.de 


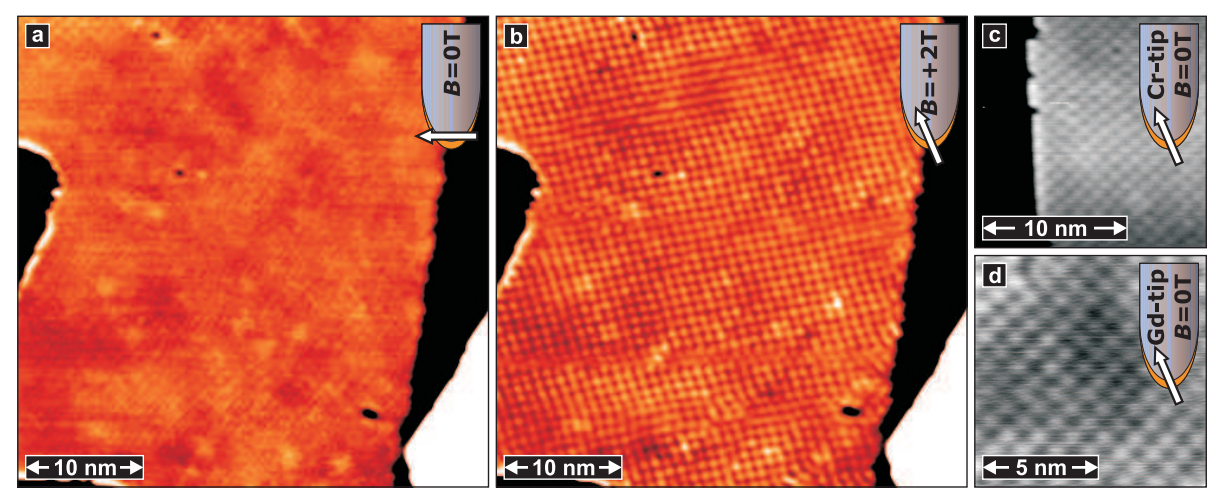

Figure 1. Fe monolayer on $\operatorname{Ir}(111)$ measured with an Fe-coated W-tip (a) without external magnetic field and (b) at the same sample area but with external field as indicated; (c), (d) measurements without applied field with out-of-plane sensitive tips.

\section{Complex magnetism of the Fe monolayer on $\operatorname{Ir}(111)$}

The system of Fe on $\operatorname{Ir}(111)$ is an interesting candidate for complex atomic scale structures: if pseudomorphic growth is achieved the Fe layer is ordered hexagonally, in contrast to the body-centered cubic structure of bulk Fe. Small precipitates of hexagonal $\mathrm{Fe}$ have been stabilized in a $\mathrm{Cu}$ matrix and were found to exhibit spin spiral structures $[6,7]$. A preparation of a hexagonal symmetry in a monolayer film allows access to the magnetic structure in real space with spin-polarized scanning tunneling microscopy $[8,9]$.

Figure 1(a) shows a monolayer area of Fe on the $\operatorname{Ir}(111)$ substrate, connected to a substrate step edge at the right side. No superstructure is observed which indicates pseudomorphic growth. Since the measurement is done with an Fe-coated W tip which is sensitive to the in-plane component of magnetization (see inset) another conclusion can be drawn from the lack of any contrast changes on the monolayer: it is either in a single ferromagnetic domain state or it has no magnetic in-plane component.

To study the out-of plane component of the sample magnetization the measurement of Fig. 1(b) was performed: a magnetic field is applied along the tip axis to change its magnetization direction (see inset). Now a superstructure becomes visible at the same sample area studied before which can directly be related to the sample magnetization: parallel to the tip magnetization at maximal intensity and antiparallel at the minima (or vice versa depending on the polarization). The observed magnetic structure has a square unit cell of about $1 \mathrm{~nm} \times 1 \mathrm{~nm}$ with one diagonal along a closed packed row. Further experiments indicate that the total magnetic moment of the structure is compensated, i.e. the amount of moments pointing up and down with respect to the sample surface is balanced. As a result the application of the external magnetic field changes the magnetization of the ferromagnetic tip but leaves the sample magnetization unaffected. To prove that the magnetic structure is not a result of the presence of an external magnetic field the measurements shown in (c) and (d) are performed with tips that are sensitive to the out-of-plane component of sample magnetization without external magnetic field. Again the same magnetic structure is observed.

From these experimental results the following magnetic structure is proposed: when assumed to be commensurable the determined unit cell holds 15 atoms as sketched in Fig. 2. We call this magnetic state mosaic structure and three rotational domains are possible. Considering the odd number of atoms in the unit cell, a balanced collinear structure is not possible when the size of the moments is constant. One possibility to construct a completely balanced structure in accordance 


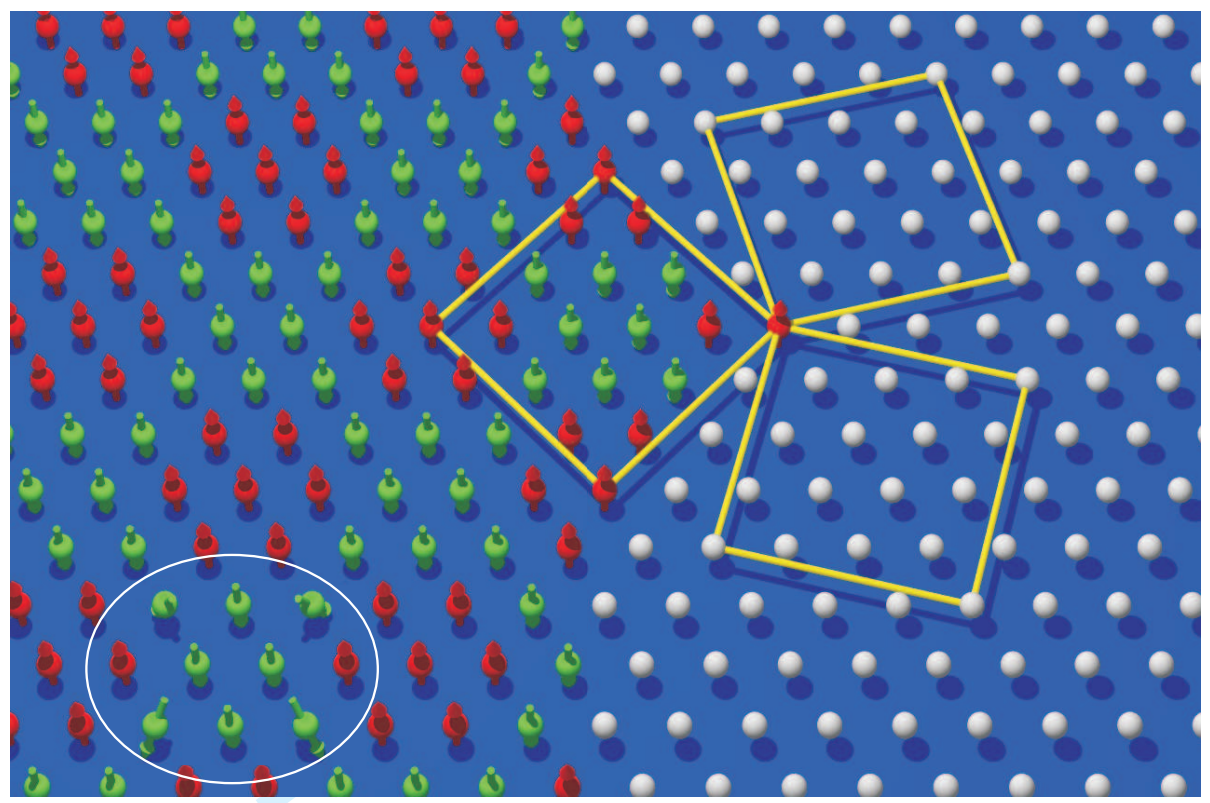

Figure 2. Mosaic structure: magnetic structure of the Fe monolayer on $\operatorname{Ir}(111)$ proposed on the basis of the experimental results. The unit cell consists of 15 atoms, with seven spins in one direction (red) and eight spins in the opposite direction (green). Three rotational domains are possible. To construct a truly balanced structure a canting of some moments is suggested as sketched exemplarily for one cell indicated by the white ellipse.

with the experimental results could be a canting of some of the moments as indicated by the white ellipse in Fig. 2. Ab-initio density functional theory calculations of the mosaic structure with this magnetic unit cell of 15 atoms show that it is lower in energy than the ferromagnetic state [8].

All three rotational domains are observed experimentally as shown in the image of Fig. 3(a). The fast Fourier transform of this image (inset) clearly shows the three sets of four spots located on a circle around the center. Spin spiral calculations as shown in (b) for the unsupported Fe monolayer and an Fe monolayer on $\mathrm{Ag}(111)$ show a typical dispersion for ferromagnetic ground states: the energy minimum is at $\bar{\Gamma}$ with a large energy difference to other magnetic states located at the high symmetry points of the Brillouin zone. In contrast to this, when Fe is on the $\operatorname{Ir}(111)$ surface, the dispersion shows an additional minimum at about $Q=$ $0.25 \times 2 \pi / a$ and the difference to other states is strongly reduced. Even though the ferromagnetic state is still the ground state within the classical Heisenberg model, the appearance of another local minimum in the dispersion is very peculiar. Interestingly, the periodicity corresponding to the local minimum is in very nice agreement with the experimentally observed magnetic unit cell, indicating that a superposition of degenerate spin spirals - a so called multi-Q state-could be the origin of the magnetic structure.

\section{Atomic spin structure of antiferromagnetic domain walls in the $\mathrm{Fe}$ mono-layer on $\mathrm{W}(001)$}

The ability of imaging antiferromagnets on the atomic scale has been demonstrated for different systems $[2,3]$. Anyhow, those structures are periodic and the question was posed, whether also non-periodic structures can be investigated with SP-STM. One possibility to induce non-periodic antiferromagnetic structures is to induce domain walls in antiferromagnets. While in bulk materials orientational domain walls have been observed with photoelectron emission microscopy with linearly polar- 

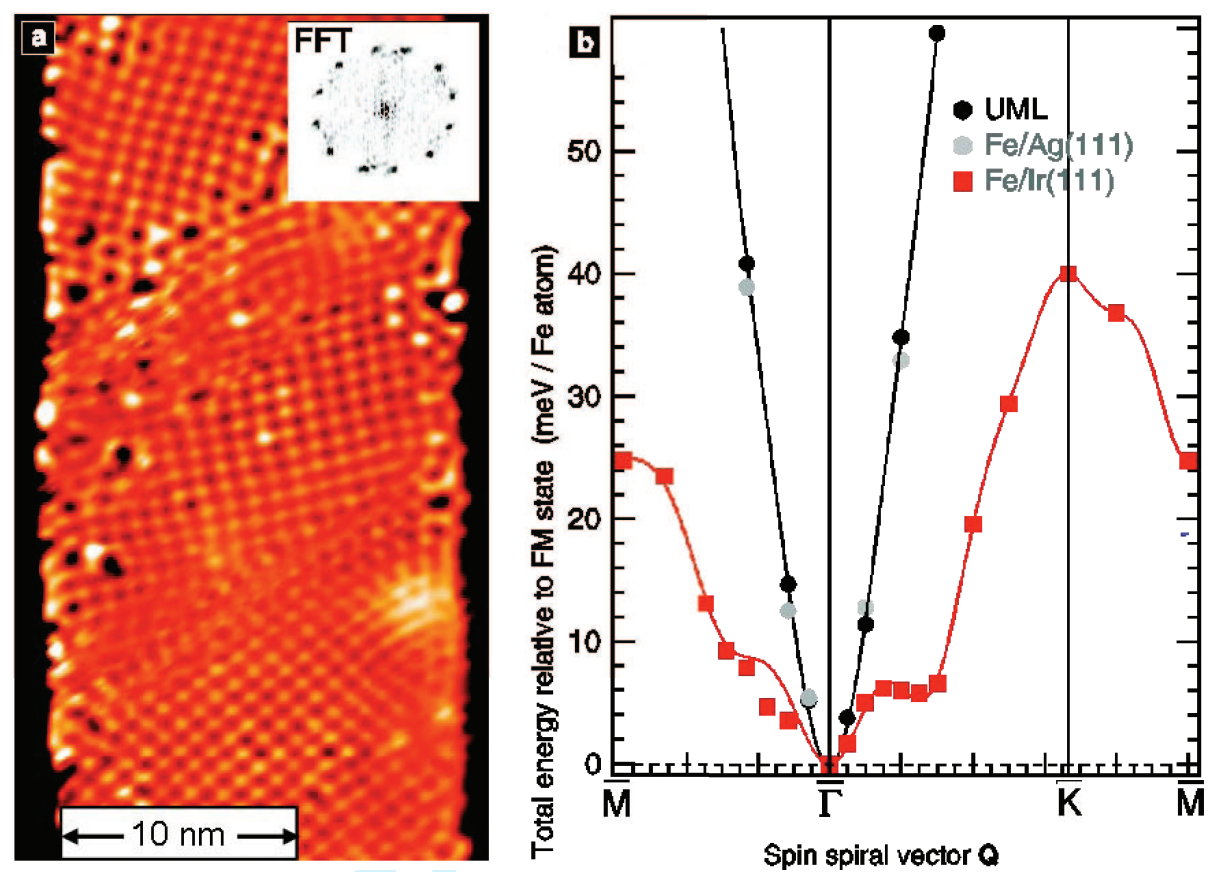

Figure 3. Magnetism of the Fe monolayer on $\operatorname{Ir}(111)$. (a) SP-STM image measured with an out-of-plane sensitive magnetic tip displaying all possible rotational domains, inset: FFT of the image. (b) Energy $E(Q)$ of flat spin spirals along high symmetry directions for an hexagonal Fe monolayer in different environments: for an unsupported Fe monolayer (UML) with the Ir lattice constant, an Fe monolayer on the $\mathrm{Ag}(111)$ surface and on the $\operatorname{Ir}(111)$ surface. Symbols mark values from first-principles calculations while the lines are a fit to the Heisenberg model including interactions up to eight nearest in-plane neighbors.

ized X-rays [10], in monolayer films with out-of-plane anisotropy no orientational domains are expected. Instead phase domain boundaries should occur, but their observation has not been reported so far, neither in thin films nor bulk materials (see Fig. 4(a)).

The system of the Fe monolayer on W(001) exhibits an out-of-plane antiferromagnetic state [3] and is thus expected to be a suitable system to induce phase domain walls [11]. Anyhow, clean monolayer samples as the one displayed in Fig. 4(b) show very large domains, and no wall could be found: one can clearly see the square lattice on the whole sample, the inset shows a closer view and a sketch of the antiferromagnetic atom arrangement. Not all atoms appear the same in the magnetic image, instead one sublattice of the antiferromagnetic structure, e.g. 'spin-up', is imaged as maximum and the other sublattice is imaged as depression. To deliberately induce walls samples which exhibit more islands of the second monolayer (ferromagnetic) are prepared to act as pinning centers for phase domain walls in the antiferromagnetic monolayer. Figure 4(c) shows a small region of such a sample with two second mono-layer islands appearing white in this representation. In the top and the bottom part of the image the square magnetic superstructure can be recognized, while the central region appears somewhat blurred. Close analysis of the position of the maxima and minima reveals that there is a phase shift between the upper and lower superstructure (see dashed white lines), i.e. that a phase domain wall is located between the second monolayer islands. Since the magnetization axis of the monolayer is out-of-plane in both domains, the magnetic moments of the wall are expected to rotate through the in-plane direction. While the image of Fig. 4(c) is imaged with a magnetic tip sensitive to the out-of-plane component of the sample magnetization, one can use a tip which is magnetized parallel to the sample surface to investigate the in-plane component of the phase domain wall. This is done in the measurement of Fig. 4(d): here one is not sensitive to the magnetic superstructure of the domains, but instead the region of the domain wall is now resolved. One 
can see, that the overall appearance resembles the antiferromagnetic superstructure which over the distance of only a few atomic rows rotates from one domain to the other. A combined ab-initio/Monte-Carlo study shows good agreement with the measured width of the domain wall. In addition it reveals that the direction of the wall is decisive for it exhibiting a stray field or not. This could be important in conjunction with exchange bias systems, where the magnetization direction of a ferromagnetic layer is pinned to an antiferromagnet.

\section{Theoretical description of antiferromagnetic domain walls in the $\mathbf{F e}$ monolayer on $\mathrm{W}(001)$}

One of the lowest exited states of antiferromagnetic system on a square lattice is a domain structure with so-called phase domains and phase domain walls (p-DW). Schematic representation of the orientational and p-DW is given in Fig. 4 . In the phase domains the checkerboard spin structure shifts laterally by one structural lattice constant. Experimentally it can only be detected indirectly by the presence of domain walls. As shown above such domain walls have been found by means of the Spin-Polarized Scanning Tunneling Microscopy (SP-STM). An exciting question is whether the phase domain walls can lead to uncompensated magnetic moments or not. This question is important as it has been postulated that a small amount of uncompensated magnetic moments at the antiferromagnet/ferromagnet interface is responsible for the exchange bias effect which is widely used in state-of-theart magnetic storage devices. The exchange bias is based on the direct exchange interaction between an antiferromagnet and a ferromagnet and leads to a signdependent magnetic coercivity of the latter. As it will be shown in the following experimental data together with Monte-Carlo simulations can infer the existence of uncompensated spins in p-DWs which are oriented along a main crystallographic direction. The uncompensated magnetization appears due to the spiral-like rotation of antiferromagnetic moments in a wall. Depending on the surface density of these p-DWs the resulting moment may lead to a significant contribution to the exchange bias.

It has been proven experimentally [3] that one atomic layer of $\mathrm{Fe}$ on $\mathrm{W}(001)$ is an antiferromagnet with the nearest and the next-nearest neighbor exchange parameters $J_{1}=20.3 \cdot 10^{-3}$ and $J_{2}=2.4 \cdot 10^{-3} \mathrm{eV} /$ atom, respectively. STM with non-magnetic tips is sensitive only to the spin-averaged local density of states (DOS) $n\left(\mathbf{r}, E_{F}\right)$ at the Fermi level (low-voltage approximation) and tip position $\mathbf{r}$. The intrinsic spin-polarization of magnetic tips, $P_{T} \equiv\left(n^{\uparrow}-n^{\downarrow}\right) /\left(n^{\uparrow}+n^{\downarrow}\right)$, where $n^{\uparrow}$ and $n^{\downarrow}$ are the majority and minority density of states, introduces a spin-polarized contribution to the tunneling current $\mathbf{I}(\mathbf{r})$ which scales with the projection of the unit vector of tip magnetization $\mathbf{u}_{T}$ onto the local magnetization DOS at $\mathbf{m}\left(\mathbf{r}, E_{F}\right)$ :

$$
\mathbf{I}(\mathbf{r}) \propto n\left(\mathbf{r}, E_{F}\right)+P_{T} \mathbf{u}_{T} \cdot \mathbf{m}\left(\mathbf{r}, E_{F}\right)
$$

This gives a magnetic contribution to constant-current mode images of periodic magnetic structures which is superimposed on the conventional topographic image. Eq. 1 can be used for calculations of a theoretical "SP-STM contrast" from the spin stucture revealed by, e.g., Monte-Carlo simulations. The internal spin structure of antiferromagnetic phase domain walls was investigated theoretically by the MC method, which is capable of simulating complex spin structures of antiferromagnets. The exchange constants $J_{1}$ and $J_{2}$ given above have been used in the calculations. Apart from the exchange interaction a perpendicu- 


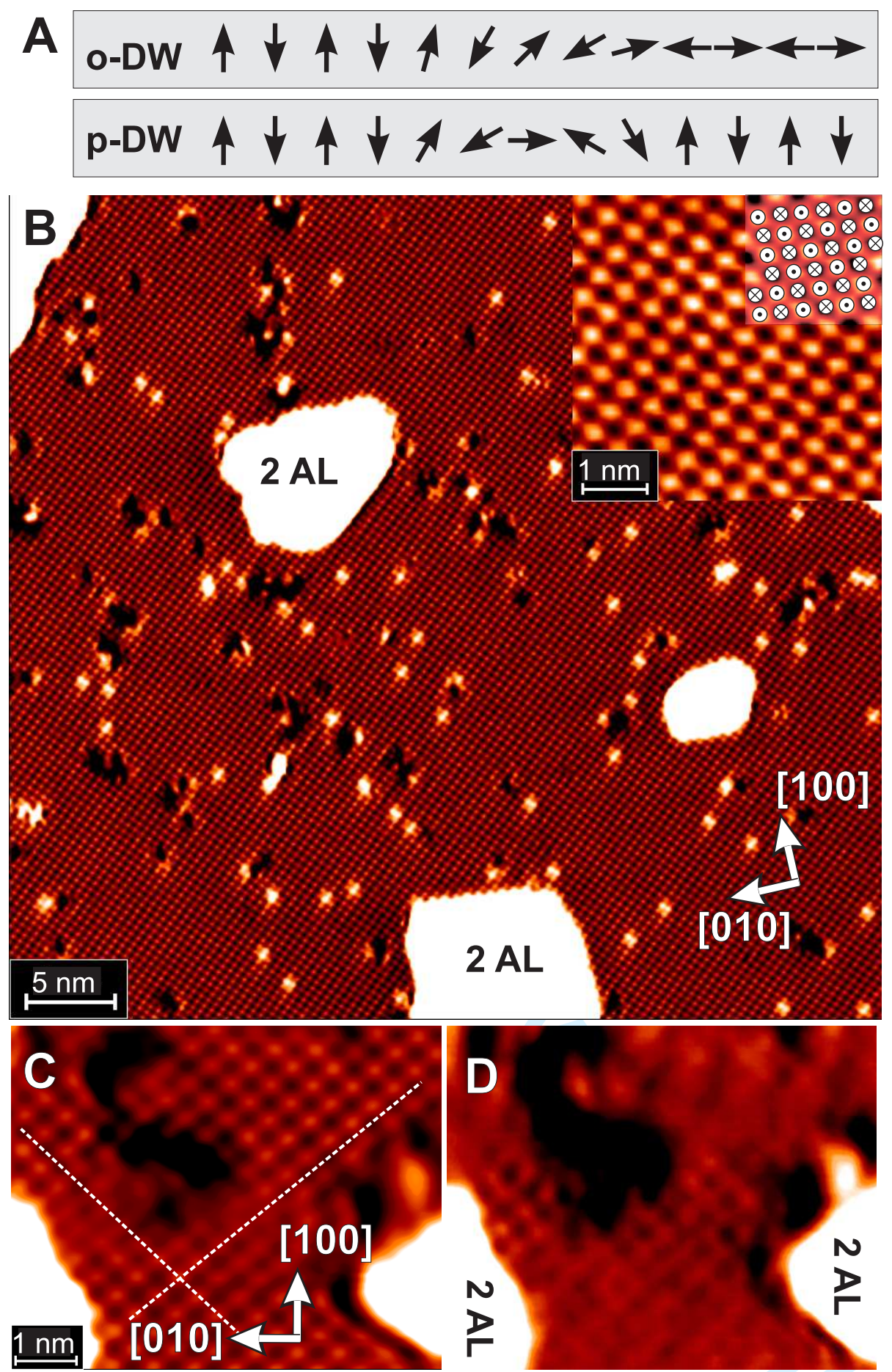

Figure 4. Schematic representation and experimental observation of domain walls (DW) at antiferromagnetic surfaces. (a) Scheme of an orientational domain wall (o-DW) and a phase domain wall (p-DW). (b) SP-STM image of 1.1 AL Fe/W(001) measured with an Fe-coated probe tip at $B=2 \mathrm{~T}$. The antiferromagnetic structure, which is shown at higher resolution in the inset, exhibits long-range periodicity without any DW visible in the field of view. p-DWs only appear at higher defect density, which can be imaged with (c) out-of-plane $(B=2 \mathrm{~T})$ and (d) in-plane sensitive tips (no field). In the constriction between the two double-layer islands, a p-DW, which runs along the [010] direction, can be seen. At the position of the p-DW, the magnetic structure shifts by one atomic site, that is, half the magnetic periodicity (see dashed lines in (c)). 
lar anisotropy and a dipolar interaction have been included into the simulations. The anisotropy energy density has been determined by ab-initio calculations to be $K_{1}=4 \mathrm{meV} /$ atom, while the strength of the magnetic dipole-dipole coupling is calculated by $D=\left(\mu_{0} g^{2} \mu_{F e}^{2}\right) /\left(4 \pi d^{3}\right)$ with $\mu_{0}$ the permeability of the vacuum, $\mu_{F e}$ the magnetic moment of antiferromagnetic iron and $d=0.3165 \mathrm{~nm}$ the interatomic Fe-Fe distance. In order to avoid that the sample eventually relaxes into a single domain state it had to be quenched rapidly from a random start configuration to the measurement temperature; i.e., $T \approx 13 \mathrm{~K}$. A characteristic result is shown in Fig. 5. The magnetic structure inside of the wall is spiral-like. No preferred orientation of the domain wall has been found. The three wall segments are oriented approximately along (a) the [110], (b) the [100], and (c) an intermediate direction. In order to compare the theoretical spin structures with experimental STM data, SP-STM images of Monte-Carlo non-collinear magnetic structures have been modeled [11]. The calculated STM images shown in Fig. 5B and C are in good qualitative agreement with the experiment: the $\mathrm{c}(2 \times 2)$ superstructure is clearly observed in the domains (domain walls) with an out-of-plane (in-plane) sensitive tip. As also found experimentally the apparent domain wall width is slightly larger for in-plane than for out-of-plane sensitive tips. This can be explained on the basis of Eq. 1: the magnetic corrugation at the domain wall scales cosine-like for an out-of-plane sensitive tip but sine-like for an in-plane sensitive tip, with the former having a rather steep zero-crossing at the domain wall position. The very weak topographical (non-magnetic) atomic contrast observed wherever $\mathbf{u}_{T} \perp \mathbf{m}_{r}$ (see, e.g. the domains in Fig. 5C) is below the experimental resolution limit.

To discuss the question whether the non-collinear p-DWs can cause uncompensated magnetic moments four principal types of p-DWs are schematically illustrated in Fig. 6: The p-DWs may be centered between (top row of Fig. 6) or on top of atomic rows (middle row) and either oriented along the [010] (left column of Fig. 6) or along the [110] direction (right column), respectively. The sketch reveals that irrespective of their symmetry [010]-oriented DWs are always compensated because adjacent spins within any row parallel to the wall point into opposite directions. The situation is different for p-DWs along [110] directions as their magnetic moments do not cancel. The direction of the uncompensated moment depends on the position of the p-DW center: if the p-DW center is on top of an atomic row it points along the spins that form the domain wall center, in the case of a wall which is centered between two atomic rows it is along the quantization axis within the domains. The bottom row of Fig. 6 shows the perpendicular component of the magnetization as obtained from MC simulations for p-DWs which almost perfectly run along the [010] (left column) and the [110] direction (right column). In agreement with the arguments mentioned above the [010]-oriented wall is compensated. Although not perfectly mirror symmetric, the domain wall center of [110]-oriented p-DWs is always found between two atomic rows and in agreement with the uncompensated moment in the simple sketch a finite perpendicular moment of about $0.6 \mu_{F e}$ per nm domain wall length remains. The azimuthal orientation of spins within the (110) wall of Fig. 5A [middle of segment (a)] is shown at a closer view in Fig. 7A. As mentioned above the MC simulations find the DW center between two atomic rows. For clarity the atomic rows are numbered successively (1)-(5) with respect to their distance from the DW center in Fig. 6A. The wall center is formed by two rows (1) with a predominant in-plane orientation $\left(65^{\circ}\right)$. With increasing distance from the DW center the moments tilt more and more into the out-of-plane direction; the in-plane component of rows (4) and (5) is already very small. Apparently, the wall is 6 to 8 atomic rows wide and Bloch-like. Comparing equidistant atomic rows located on opposite sides of the DW center it becomes 


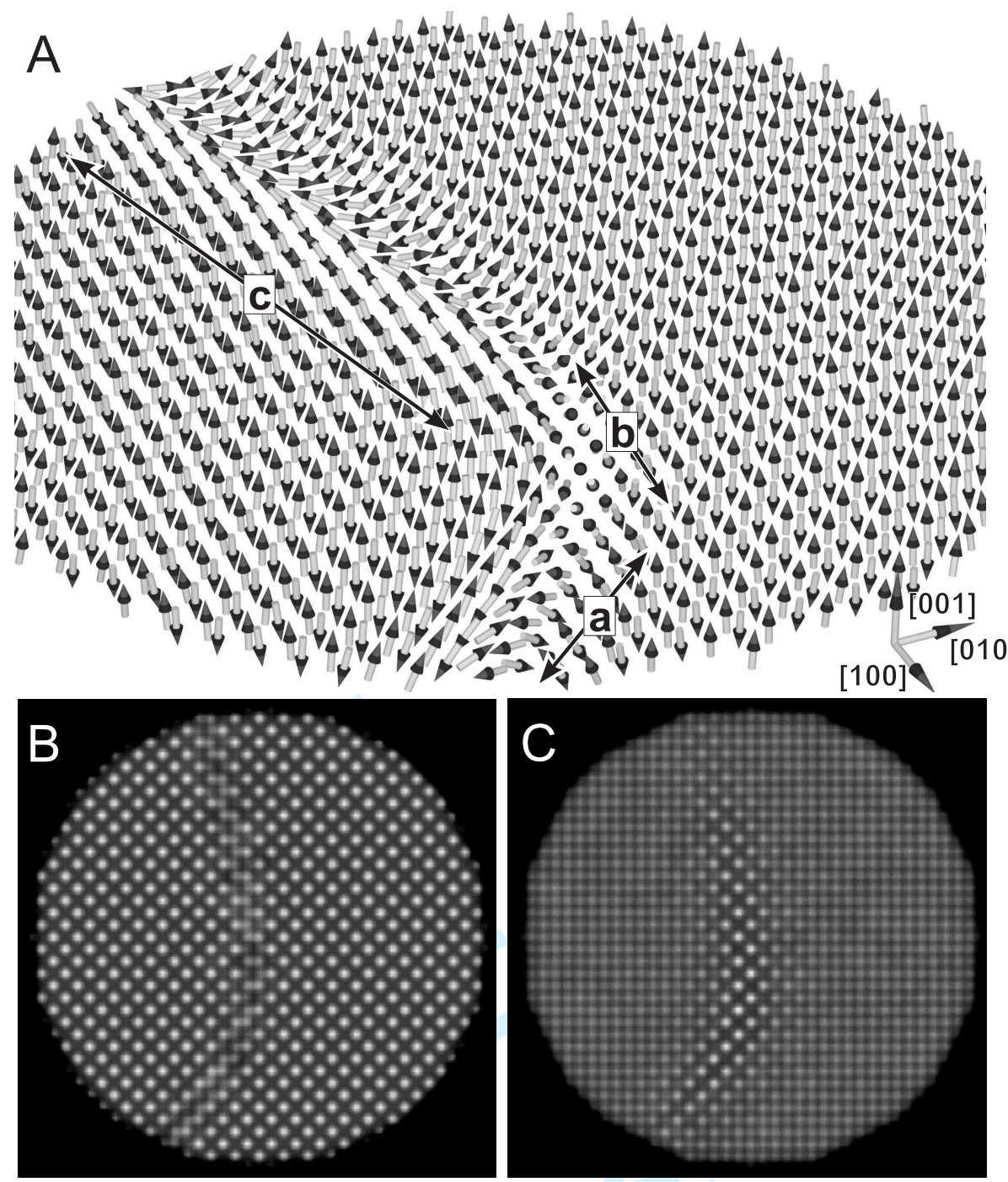

Figure 5. Monte-Carlo (MC) simulation of antiferromagnetic domain walls. (A) Rendered perspective image of the quenched spin structure of an antiferromagnetic material as obtained by MC simulation. A domain wall can be recognized which exhibits three different orientations: (a) along the [110], (b) [100], and (c) an intermediate direction. Calculated SP-STM images for (B) out-of-plane and (C) in-plane sensitive magnetic tips.

clear that the in-plane component is reversed while the out-of-plane component is equal. Thereby, the integrated in-plane component of magnetization is perfectly canceled but, interestingly, a non-vanishing net magnetic moment remains for the out-of-plane component.

Due to the fact that domain walls in antiferromagnets cost exchange energy but cannot lower the dipolar energy they are very rare and short on clean surfaces. It is, however, likely that they may be much more frequent in a typical exchange-bias situation. Here, the antiferromagnet is covered with a ferromagnetic film which typically exhibits a higher magnetic ordering temperature than the antiferromagnet. Consequently, the antiferromagnet is in contact with a ferromagnet when it orders magnetically. It is expected that in this case the exchange coupling to the ferromagnet induces a relevant number of p-DWs. 


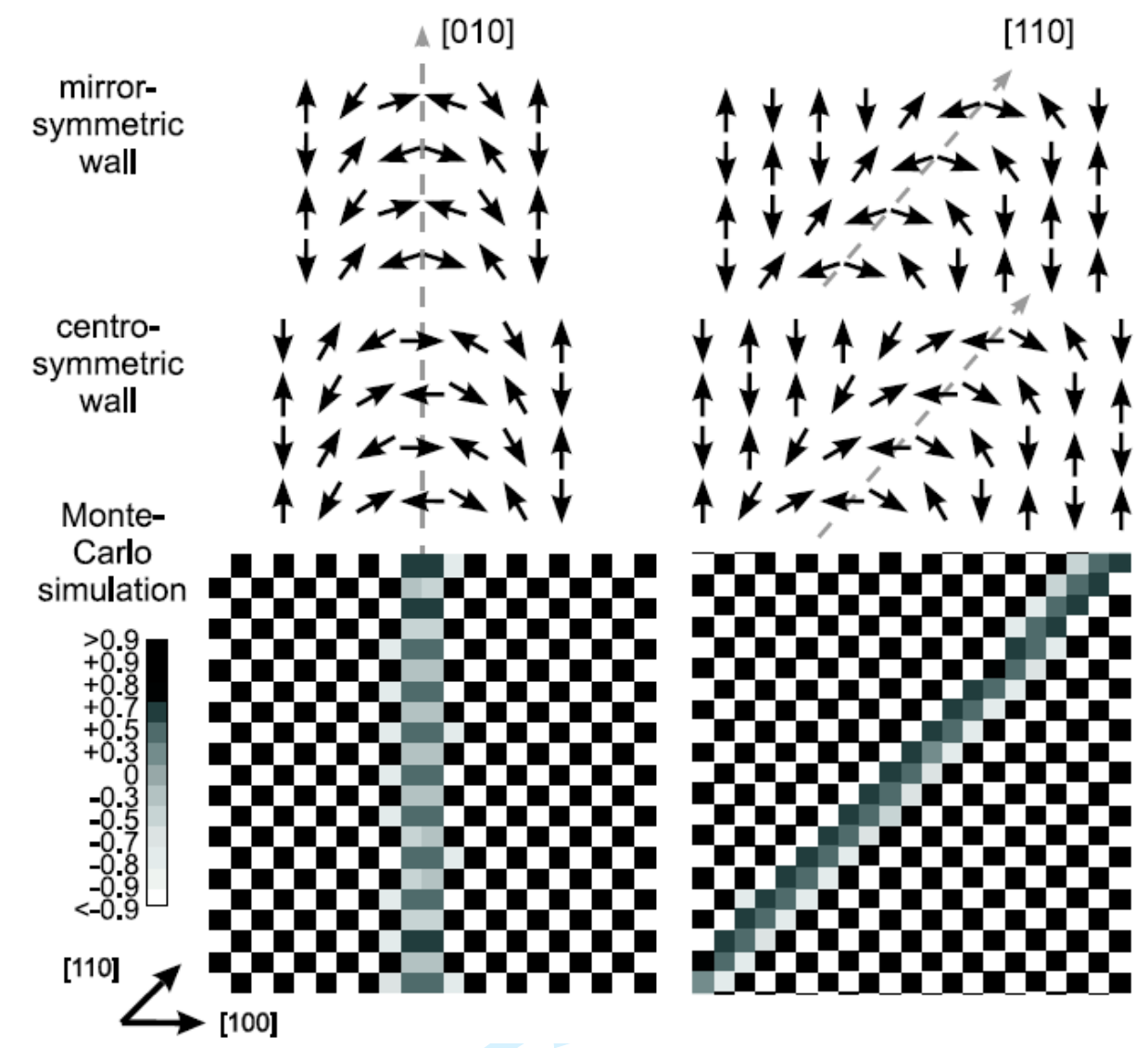

Figure 6. Spin configuration of [010]- and [110]-oriented domain walls. Schematic representation of p-DWs which are centered between (top row) and on top of atomic rows (middle row). (bottom row) Part of a p-DW oriented along the [010] (left) and the [110] direction (right) from Monte-Carlo simulations. The gray scale gives the calculated out-of-plane component of the magnetization.
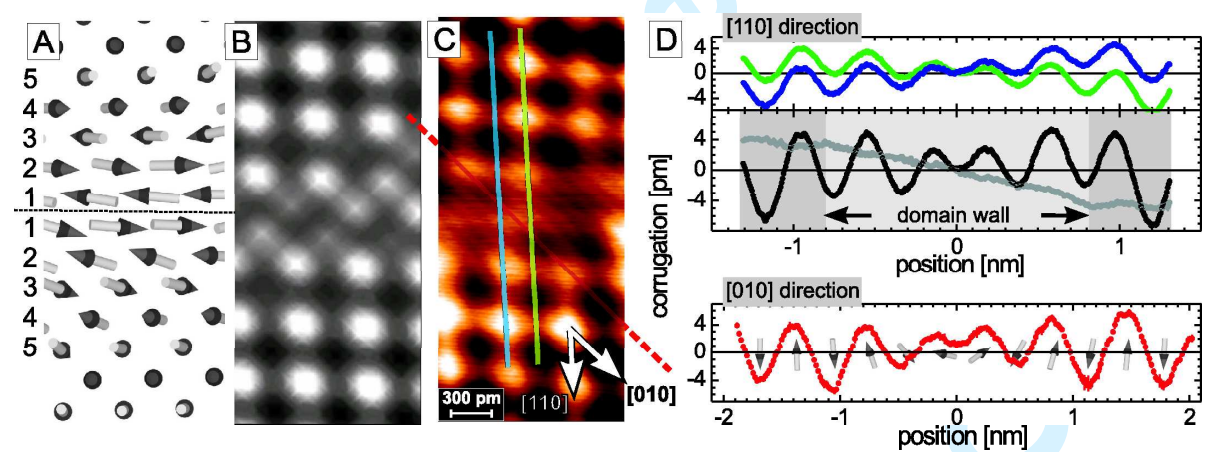

Figure 7. Detailed view of a [110]-oriented spiral p-DW. (A) Theoretical spin structure, (B) simulated, and (C) experimental SP-STM image of a p-DW in the out-of-plane antiferromagnetic Fe monolayer on $\mathrm{W}(001)$. (D) Height profiles drawn at the positions of the correspondingly colored lines in (C) along the [110] (upper panel) and the [010] direction (lower panel). Middle panel: Sum (black) and difference (gray) of the line profiles shown in the upper panel. The wall is about $1.6 \mathrm{~nm}$ wide and its out-of-plane component exhibits mirror symmetry.

5. Observation of a spin spiral state in the Mn monolayer on $\mathrm{W}(110)$

The system of a Mn monolayer on W(110) has been investigated previously with SP-STM and ab-initio calculations and local antiferromagnetic order was found [2]. Recent measurements on a larger scale are shown in Fig. 8: while the vertical stripes in the spin-resolved images (left) are clear indications for the antiferromagnetic order, they nearly vanish periodically about every $5 \mathrm{~nm}$. Again measurements with differently magnetized tips at the same sample position reveal the origin of 

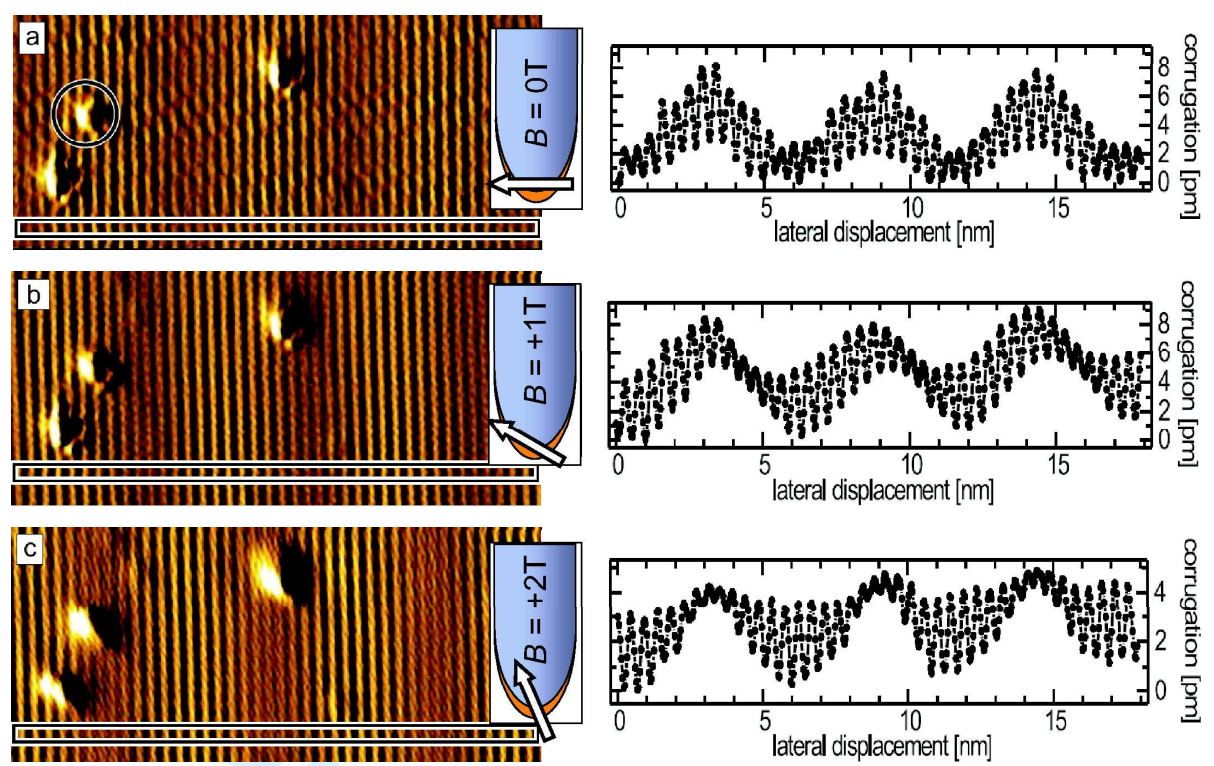

Figure 8. Field-dependent SP-STM measurements. Magnetically sensitive constant-current images of the Mn monolayer on W(110) (left panels) and corresponding line sections (right panels) taken with a ferromagnetic Fe-coated tip at external fields of $0 \mathrm{~T}(\mathrm{a}), 1 \mathrm{~T}(\mathrm{~b})$ and $2 \mathrm{~T}$ (c). As sketched in the insets, the external field rotates the tip magnetization from in-plane (a) to out-of-plane (c), shifting the position of maximum spin contrast.

this effect: while the tip used in (a) -magnetized parallel to the sample surfacereveals the antiferro-magnetic arrangement of in-plane moments, the tip in (c) is magnetized perpendicular to the surface and shows the stripe pattern whereever the magnetic moments of the sample are out-of-plane. Clearly a comparison between (a) and (c) shows that the area where the magnetic superstructure dominates the image shifts, and the measurement of (b) with intermediate tip magnetization angle shows that the maximum amplitude of the stripes propagates to the left in this measurement. These observations prove that the Mn monolayer on W(110) has locally an antiferromagnetic structure, which rotates to form a spin spiral state along the [110] direction [12]. Two possible mechanisms for the formation of a spin spiral come to mind: first, a competition of exchange interactions between atoms, e.g. antiferromagnetic nearest neighbor coupling and also antiferromagnetic nextnearest neighbor coupling, or a twist between adjacent magnetic moments due to Dzyaloshinskii-Moriya interaction [13, 14], which is a result of spin-orbit interaction in an environment lacking inversion symmetry. While an exchange driven spin spiral can rotate either way and leads to both rotational senses, a spin spiral that is induced by Dzyaloshinskii-Moriya interaction favors one rotational sense and will never form a spiral with the opposite rotation.

To investigate the origin of the spin spiral in the Mn monolayer on W(110) the energy of spin spirals is calculated as a function of the periodicity, see Fig. 9 (left, with the two main crystallographic directions in upper and lower part, respectively). When spin orbit coupling is neglected the minimum in energy is at the high symmetry N point (crosses in Fig. 9 (left)), which corresponds to the collinear $\mathrm{c}(2 \times 2)$ antiferromagnetic state, which was proposed before [2]. This finding indicates, that competing exchange interactions cannot be responsible for the spin spiral formation, since in that case two symmetric minima at $\lambda>0$ would be expected. To find out whether Dzyaloshinskii-Moriya interaction is really responsible for the twist between adjacent moments spin-orbit coupling was introduced into the calculation as a perturbation. Now the minimum shifts to lower energy and to only one side of the high symmetry point (dots in Fig. 9 (left)) which indeed shows that due to the Dzyaloshinskii-Moriya interaction the energy for a spin spiral with 

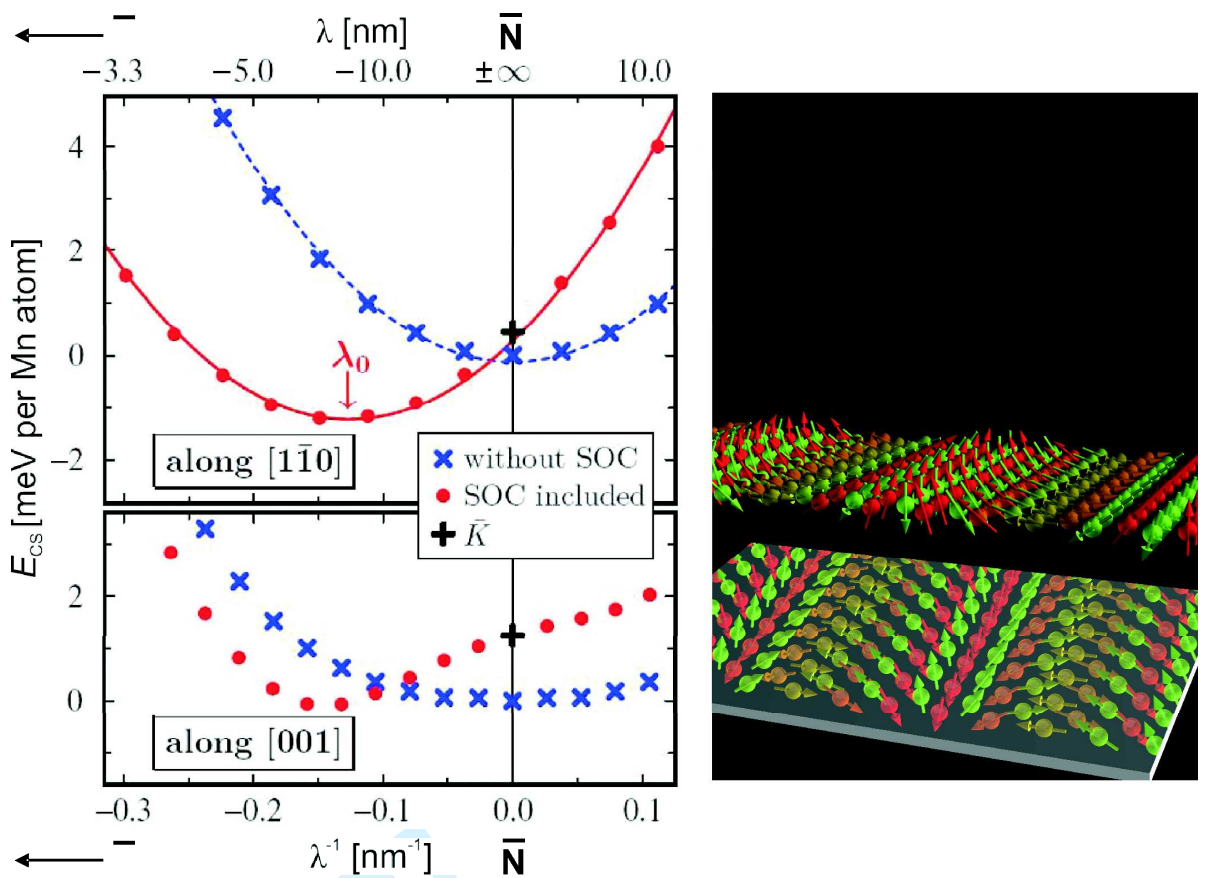

Figure 9. Left: Calculated energy of homogeneous cycloidal spin spirals propagating along the two highsymmetry directions [110] (upper panel) and [001] (lower panel) as function of the period length, $|\lambda|$. Origin of energy is the $c(2 \times 2)$ antiferromagnetic state. The data points show the density functional theory results obtained without (blue crosses) and with (red dots) spin-orbit coupling (SOC) included. Broken $(D=\bar{K}=0)$ and solid lines are fits to the energy of a cycloidal spiral. Note that the possible energy gain is much larger for propagation along the [110] direction than along the [001] direction. Right: shows a perspective view of an antiferromagnetic cycloidal spin spiral; in the mirror one can see the opposite rotational sense of such a spiral (courtesy of P. Ferriani).

a specific rotational sense is lowered compared to the $\mathrm{c}(2 \times 2)$ antiferromagnetic state. The right part of Fig. 9 shows a perspective view of such a cycloidal spin spiral, and the reflection in the mirror at the lower part of the image mimics the other rotational sense which is higher in energy.

\section{Chiral magnetic ordering in two-dimensional ferromagnets with competing Dzyaloshinskii-Moriya interactions}

Single crystals of magnetic materials exhibit periodicities on at least two different length scales - that of the atomic lattice, and that of the magnetic structure. Such systems, which consist of a perfectly periodic crystal lattice and an additional periodic modulation of some order parameter, are denoted as modulated structures. In centrosymmetric, magnetically ordered crystals the handedness of modulated structures is energetically degenerate. However, if the inversion symmetry is broken for some reasons, this degeneracy may be lifted because the electronic spin-orbit scattering induces chiral asymmetry of exchange coupling. In such noncentrosymmetric systems an additional order parameter - chirality - might appear. The chiral ordering is very interesting from the theoretical point of view as well as for technical applications.

It has been shown recently that antisymmetric exchange interactions first predicted by Dzyaloshinskii [13] can be especially strong near magnetic surfaces and in nanostructures due to reduced symmetry and large strains. The directions of Dzyaloshinskii-Moriya (DM) vectors have been investigated for several surfaces [15] and the existence of non-collinear magnetic structures in thin films has been postulated. Existing studies of chiral magnetic ordering have been limited up to 
now to one-dimensional chiral structures with a single DM vector or to frustrated bulk pyrochlores. The evolution of magnetic nano-ordering in mono/double layers as a function of length and orientation of several DM vectors has not been investigated so far.

The description of magnetic ordering in two-dimensional systems with several DM vectors is not trivial for several reasons. First, for many lattice symmetries the orientation and the strength of the DM interactions cannot be determined using the theoretical concepts of Ref. $[13,15]$. Second, different DM couplings may compete, and third, the influence of the magnetic anisotropy on the chiral ordering is not known. Magnetic order on the nanoscale, however, is very important for the description of hysteretic and dynamic properties of nano-objects. The aim of our investigation is to achieve a spatially resolved description of the magnetization patterning in two-dimensional lattices in the presence of competing DM interactions and a magnetocrystalline anisotropy.

In order to explore the intriguing question as to what extent the competition between several Dzyaloshinskii-Moriya vectors affects the magnetization patterning, we performed Monte-Carlo (MC) simulations for samples of up to $200 \times 200$ classical spins on two-dimensional lattices with open and periodic boundary conditions [16]. Square, rectangular, 'losenge' and triangular symmetries have been chosen for the calculations as they correspond to different surfaces of sc, bcc, and fcc parent structures for which non-negligible DM interactions have been predicted [15]. Additionally, we have performed calculations for three-dimensional Fe/W(110) nanowires. However, it has been assumed that the DM interactions have a nonvanishing amplitude only at the surface atomic layer of a nanowire.

The classical spin-lattice Hamiltonian reads

$$
\begin{array}{r}
H=\sum_{i<j} J\left(\vec{R}_{i j}\right) \vec{S}_{i} \cdot \vec{S}_{j}+ \\
+\sum_{i, j} \vec{D}_{\vec{R}_{i j}}^{\vec{n}} \cdot\left(\vec{S}_{i} \times \vec{S}_{j}\right)+K(\vec{m}) \sum_{i}\left(\vec{S}_{i} \cdot \vec{m}\right)^{2} \\
+d \sum_{i<j}\left(\frac{\vec{S}_{i} \cdot \vec{S}_{j}}{R_{i j}^{3}}-3 \frac{\left(\vec{S}_{i} \cdot \vec{R}_{i j}\right)\left(\vec{S}_{j} \cdot \vec{R}_{i j}\right)}{R_{i j}^{5}}\right)
\end{array}
$$

where $\vec{S}_{i}$ is a three-dimensional unit spin vector at a site $i ; \vec{R}_{i j}=\vec{R}_{i}-\vec{R}_{j}$ the distance-vector between the $i^{\text {th }}$ and the $j^{\text {th }}$ site and the set $\left\{\vec{R}_{i}\right\}$ defines a threedimensional lattice $L^{(3)}$. The vectorial DM interaction $\vec{D}_{\vec{R}_{i j}}^{\vec{n}}$ depends on both the distance vector $\vec{R}_{i j}$ and the orientation $\vec{n}$ of the DM vector in the three-dimensional space $O^{(3)}$. It has been found in our simulations that periodicity of a spiral $P$ is determined by the competition between the exchange and the DM interaction energy. Hence, $P$ increases with a decreasing ratio $\left|\vec{D}_{\vec{R}_{i j}}^{\vec{n}}\right| / J$. The log-log plot reveals a power law $P \propto\left(\left|\vec{D}_{\vec{R}_{i j}}^{n_{1}}\right| / J\right)^{-\beta(D / J)}$ for all types of studied lattices. In addition, for identical $\left|\vec{D}_{\vec{R}_{i j}}^{\vec{n}_{i j}}\right| / J$ ratio the periodicity of the DM spirals propagating along high symmetry directions of a lattice is somewhat larger than those along other directions because of the competition between the DM- and the strong exchange interaction. In these calculations only first nearest neighbors have been taken into account. 

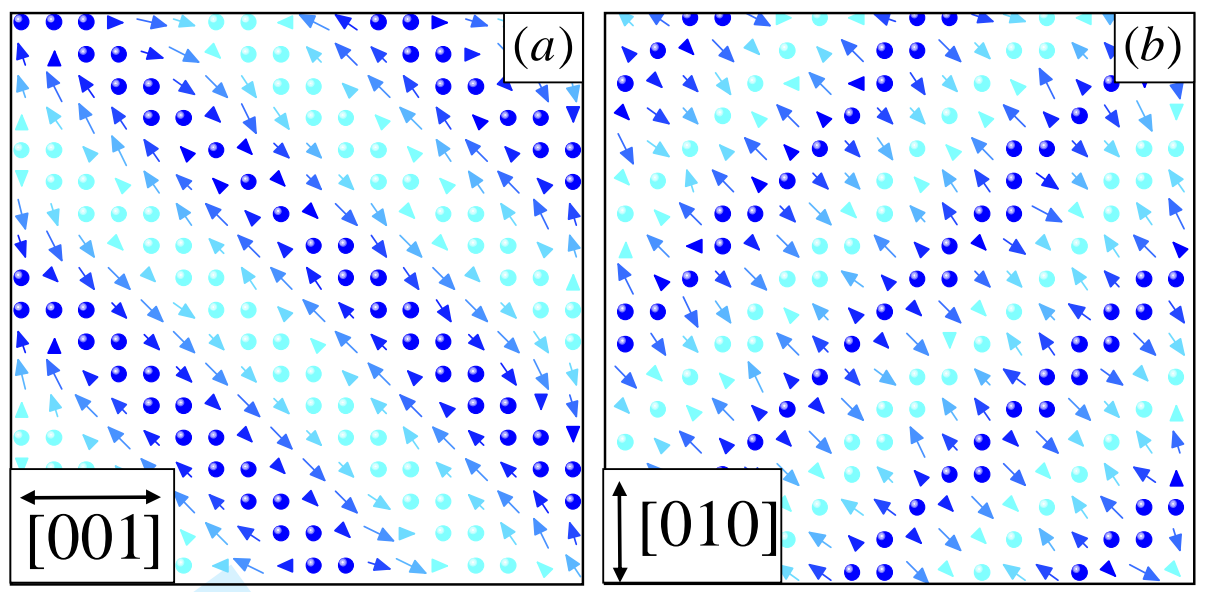

Figure 10. Top view of portions of stable MC configurations for a simple cubic lattice corresponding to a free standing bcc(001) monolayer with a ferromagnetic exchange interaction. (a) $\left|\vec{D}_{[100]}^{[010]}\right|=0.8 J=$ $\left|\vec{D}_{[010]}^{[100]}\right| ;(\mathrm{b})\left|\vec{D}_{[100]}^{[010]}\right|=0.8 J=-\left|\vec{D}_{[010]}^{[100]}\right| ; k T / J=0.05$. The insets at the bottom of (a) and (b) give averaged magnetization profiles along [001] direction for the two cases. The color scheme denotes the vertical component of magnetization $S_{z}$.

In the next step the influence of the magnetic anisotropy $\vec{K}$ on a chiral ordering has been studied. The tendency is the following: when the anisotropy axis lies within the plane of magnetization rotation defined by the DM coupling the anisotropy favors the increase of the width of parts of the spiral parallel to $\vec{K}$. In other words a domain structure or an inhomogeneous spiral is formed. If $\vec{K}$ is perpendicular to the rotation plane it destroys the spiral already for very small $K / J \geq 0.1\left|\vec{D}_{\vec{R}_{i j}}^{\vec{n}}\right|$ ratios.

For several non-vanishing DM vectors we find that the magnetic ground state is a superposition of spirals. This superposition is non-trivial as quite a few configurations depending on the phase, strength and sign of the initial spirals are possible. In addition the lattice symmetry and the strength of the exchange interaction play a very important role. For example, for the calculations on a square lattice we considered two perpendicular DM vectors. One of them propagates along the [100] axis and is oriented parallel to [010] while another one runs along the [010] direction and is of [100] orientation.

If both DM vectors are of identical length the propagation direction $\vec{R}_{p}$ of the modulated structure forms an angle of 45 degrees with respect to each axis of high symmetry (see Fig. 10). There are two possibilities for such an orientation: [110] and [110]. The two directions are energetically degenerate. Apparently the same should be true for the orientation of magnetization. For $\left|\vec{D}_{[100]}^{[010]}\right|=\left|\vec{D}_{[010]}^{[100]}\right|$ the magnetic moments are oriented with an angle of $45^{\circ}$ with respect to the principal axes. However, for each direction of propagation characterized by a vector $\vec{R}_{p}$, two magnetization orientations are possible: parallel or perpendicular to $\vec{R}_{p}$. Our calculations demonstrate that a sign of the DM interaction plays a crucial role for the orientation of magnetization. The sign of each DM vector determines the chirality of a spiral. For a single DM vector the spirals with opposite chirality have identical energy, but the energy of a superimposed state depends on the chirality of each constituent. While for $\left|\vec{D}_{[100]}^{[010]}\right|=\left|\vec{D}_{[010]}^{[100]}\right|$ and $\vec{D}_{[100]}^{[010]} \cdot[010]=\vec{D}_{[010]}^{[100]} \cdot[100]$ a Bloch like rotation is energetically favorable (see Fig. $10(\mathrm{a})$ ), for $\left|\vec{D}_{[100]}^{[010]}\right|=\left|\vec{D}_{[010]}^{[100]}\right|$ with $\vec{D}_{[100]}^{[010]} \cdot[010]=-\vec{D}_{[010]}^{[100]} \cdot[100]$ the Néel type of rotation applies (see Fig. 10 (a)). It is, however, worth mentioning that because of the symmetry considerations the Bloch 

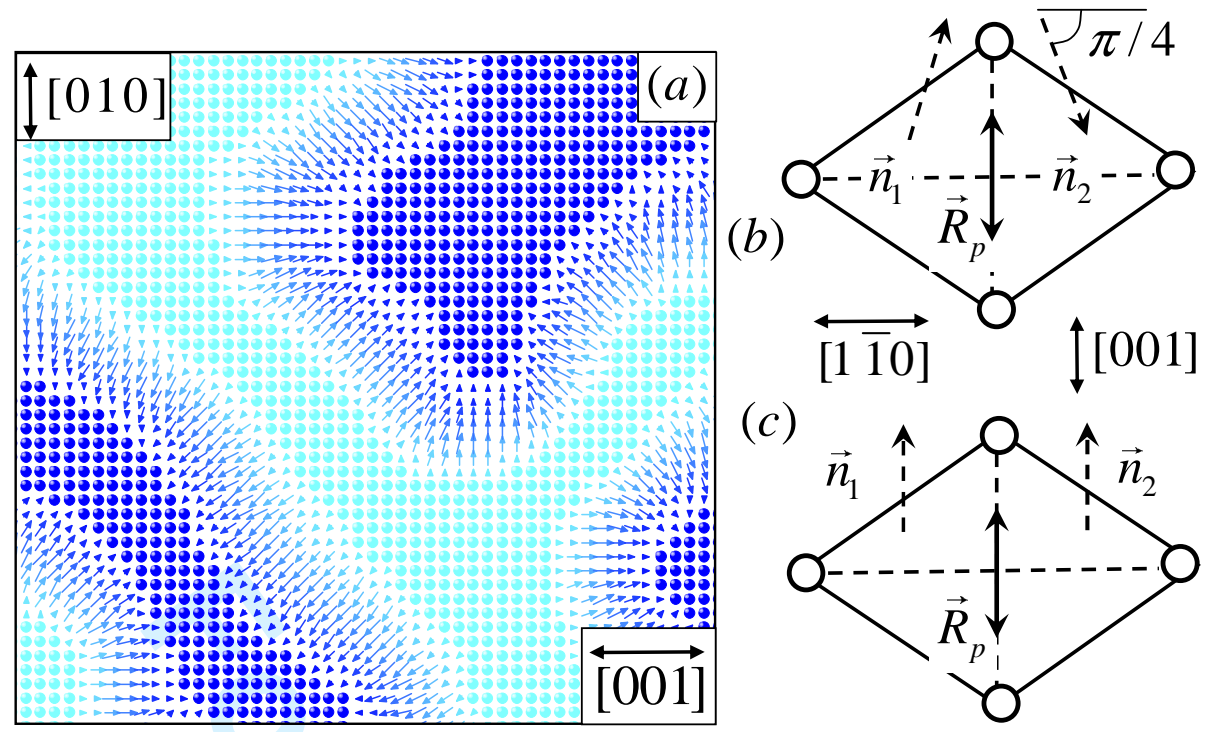

$(c)$

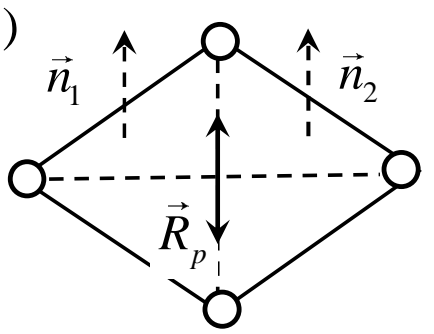

Figure 11. (a) Top view of a portion of a domain wall between energetically degenerated regions of a periodic DM modulation on a square lattice corresponding to a free standing bcc $(001)$ monolayer with a ferromagnetic exchange interaction. The system is characterized by two DM vectors: $\left|\vec{D}_{[100]}^{[010]}\right|=-\left|\vec{D}_{[010]}^{[100]}\right|=$ $0.2 J ;$ (b) and (c) Schematic representation of the orientation of DM vectors leading to the perfect chirality of [110] domain walls in $\mathrm{Fe} / \mathrm{W}(110)$ with (b) a Néel and (c) a Bloch rotation of magnetization.

type of the spiral $\left(\vec{D}_{[100]}^{[010]} \cdot[010]=\vec{D}_{[010]}^{[100]} \cdot[100]\right)$ is not possible in solids consisting of one type of atoms only.

Because of the energetic degeneracy of the two propagation directions on (100) surfaces we find domain formation in samples with DM interactions. The energy cost for domain walls is small ( $<2 \%$ of the total energy) for realistic values of $\left|\vec{D}_{\vec{R}_{i j}}^{\vec{n}}\right|$ as a very smooth transition from one spiral orientation to the other is possible. A typical domain wall generated by the Monte-Carlo procedure on a square lattice is shown in Fig. 11 (a).

\section{Chirality of domain walls in $\mathrm{Fe} / \mathrm{W}(110)$}

Fe layers on a stepped W(110) substrate have been extensively studied experimentally and theoretically $[17,18]$. In the coverage regime between one and two monolayers of $\mathrm{Fe}$, the system is characterized by alternating monolayer and double layer stripes. Both regions show a periodic magnetic domain structure. The periodicity of the pattern as well as the width of mono/double layer regions depends on the Fe coverage. The typical distance between adjacent walls for the coverage of 1.7 monolayers is of the order of $20 \mathrm{~nm}$. The domains are separated by $180^{\circ}$ domain walls which always run along the [1ํㅣㄹ direction. While in monolayers domains with two opposite in-plane orientations have been observed, in double layers out-of-plane domains alternatingly magnetized up and down exist [17, 18]. Experimentally a perfect chirality of magnetic domain walls for the whole area of a large sample has been reported. This finding has never been supported by theoretical studies although all other parameters like the orientation of domain walls or the domain size have been described theoretically [19].

We have demonstrated that the DM interaction is responsible for the perfect chirality of magnetic domain walls in $\mathrm{Fe} / \mathrm{W}(110)$ [16]. It follows from our calculations that there is a manifold of modulated magnetic structures depending on the relative orientation and the modulus of $\vec{n}_{1}$ and $\vec{n}_{2}$. However, only some solutions support the experimentally observed magnetic domain structure. Two possibilities 
are schematically shown in Fig. 11 (b), (c). The situation in Fig. 11 (b) corresponds to domain walls of Néel type, while that of Fig. 11 (c) to the Bloch type. In both cases the walls are running along $[1 \overline{1} 0] \perp \vec{R}_{p}$. Most recent experiments performed for Fe double layers on W(110) support the existence of Néel-type walls.

\section{Acknowledgements}

We would like to thank M. Heide, G. Bihlmayer, S. Blügel, P. Ferriani, S. Heinze, L. Udvardi, and P. Weinberger for their contributions to the theoretical understanding of our experimental SP-STM data.

Financial support of the Deutsche Forschungsgemeinschaft (SFB 668), the Stifterverband für die Deutsche Wissenschaft and the Interdisciplinary Nanoscience Center Hamburg (INCH) is gratefully acknowledged.

\section{References}

[1] R. Wiesendanger, I. V. Shvets, D. Bürgler, D. Tarrach, H.-J. Güntherodt, J. M. D. Coey, and S. Gräser, Science 255 (1992), p. 583.

[2] S. Heinze, M. Bode, A. Kubetzka, O. Pietzsch, X. Nie, S. Blügel, and R. Wiesendanger, Science 288 (2000), p. 1805

[3] A. Kubetzka, P. Ferriani, M. Bode, S. Heinze, G. Bihlmayer, K. von Bergmann, O. Pietzsch, S. Blügel, and R. Wiesendanger, Phys. Rev. Lett. 94 (2005), p. 087204.

[4] M. Pratzer, H. J. Elmers, M. Bode, O. Pietzsch, A. Kubetzka, and R. Wiesendanger, Phys. Rev. Lett. 87 (2001), p. 127201.

[5] S. Heinze, Appl. Phys. A 85 (2006), p. 407.

[6] Y. Tsunoda, J. Phys.: Condens. Matter 1 (1998), p. 10427.

[7] K. Knöpfle, L. M. Sandratskii and J. Kübler, J Phys. Rev. B 62 (2000), p. 5564.

[8] K. von Bergmann, S. Heinze, M. Bode, E. Y. Vedmedenko, G. Bihlmayer, S. Blügel, and R. Wiesendanger, Phys. Rev. Lett. 96 (2006), p. 167203.

[9] K. von Bergmann, S. Heinze, M. Bode, G. Bihlmayer, S. Blügel, and R. Wiesendanger, New Journ. Phys. 9 (2007), p. 396.

[10] N. B. Weber, H. Ohldag, H. Gomonaj and F. U. Hillebrecht, Phys. Rev. Lett. 91 (2003), p. 237205.

[11] M. Bode, E. Y. Vedmedenko, K. von Bergmann, A. Kubetzka, P. Ferriani, S. Heinze, and R. Wiesendanger, Nature Materials 5 (2006), p. 477.

[12] M. Bode, M. Heide, K. von Bergmann, P. Ferriani, S. Heinze, G. Bihlmayer, A. Kubetzka, O. Pietzsch, S. Blügel, and R. Wiesendanger, Nature 447 (2007), p. 190.

[13] I. Dzyaloshinskii, Sov. Phys. JETP 5 (1957), p. 1259.

[14] T. Moriya, Phys. Rev. 120 (1960), p. 91.

[15] A. Crépieux and C. Lacroix, J. Magn. Magn. Mater. 182 (1998), p. 341.

[16] E. Y. Vedmedenko, L. Udvardi, P. Weinberger, and R. Wiesendanger, Phys. Rev. B 75 (2006), p. 104431.

[17] A. Kubetzka, M. Bode, O. Pietzsch, and R. Wiesendanger, Phys. Rev. Lett. 88 (2002), p. 057201.

[18] A. Kubetzka, O. Pietzsch, M. Bode, and R. Wiesendanger, Phys. Rev. B 67 (2003), p. 20401.

[19] E. Y. Vedmedenko, A. Kubetzka, K. von Bergmann, O. Pietzsch, M. Bode, H. P. Oepen, J. Kirschner and R. Wiesendanger, Phys. Rev. Lett. 92 (2004), p. 077207. 


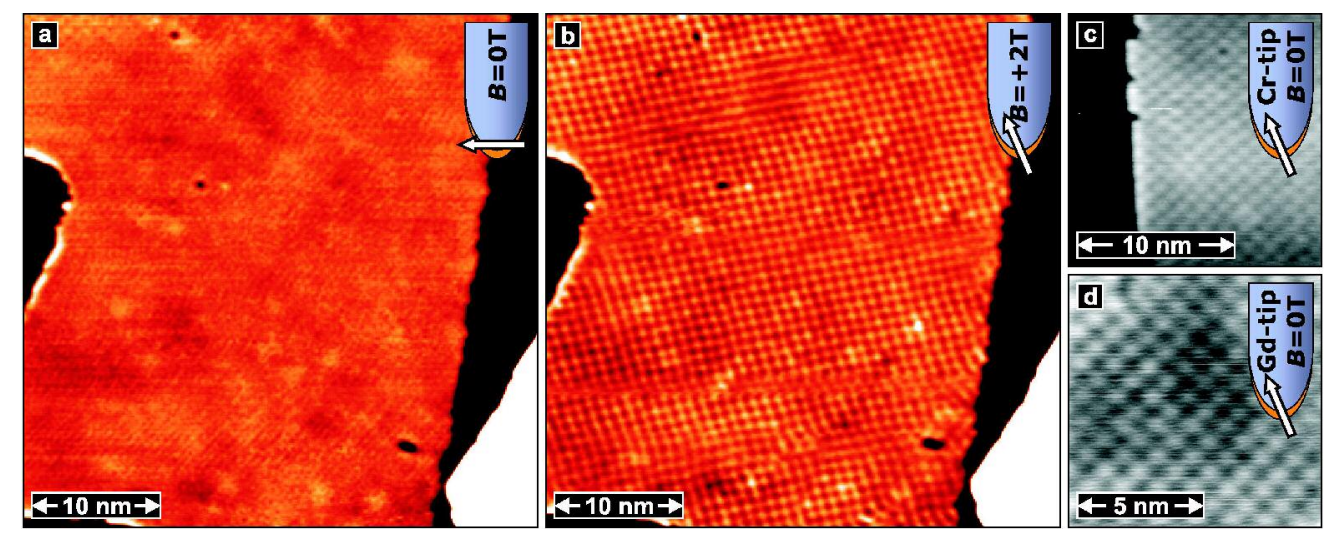

$152 \times 60 \mathrm{~mm}(600 \times 600$ DPI $)$ 


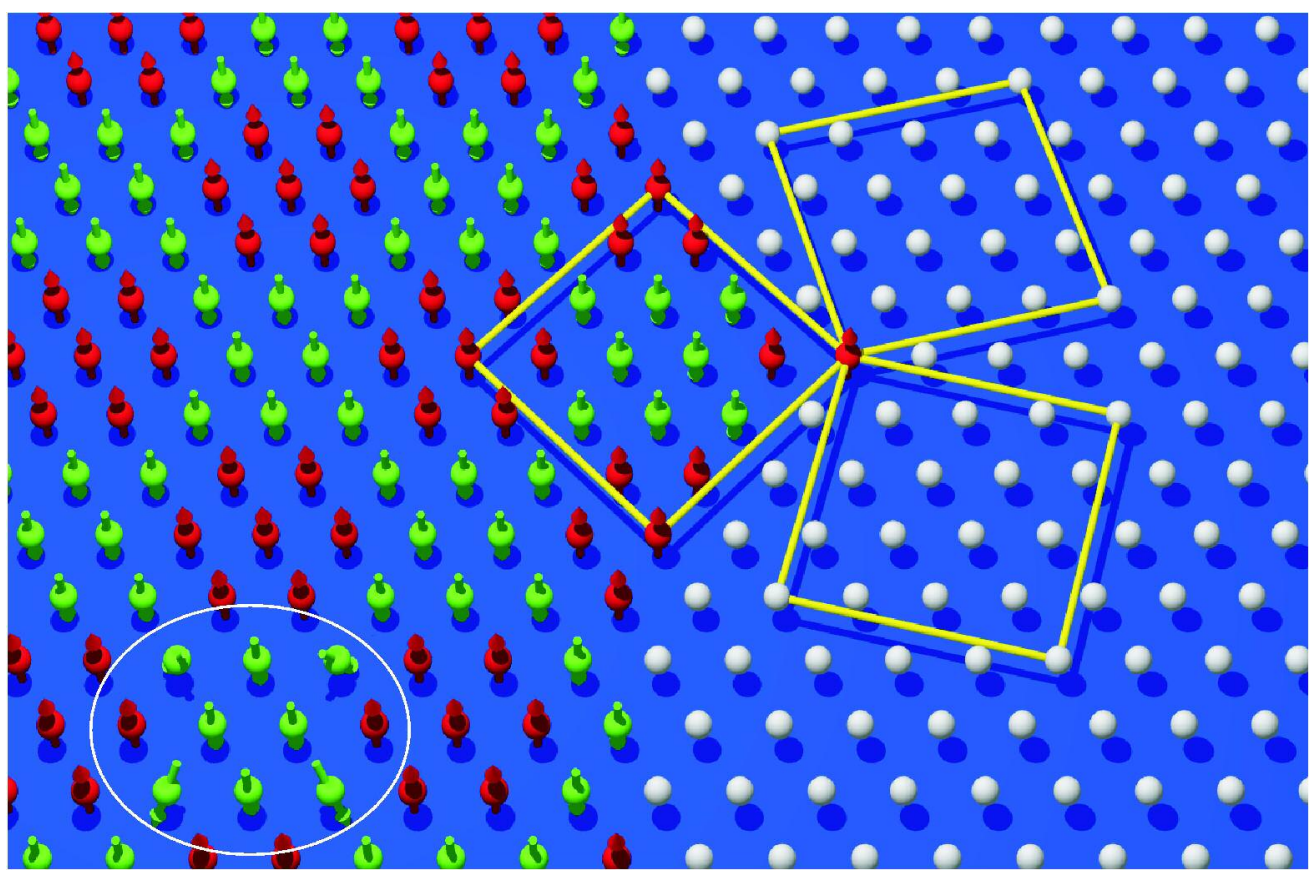

$180 \times 119 \mathrm{~mm}(600 \times 600 \mathrm{DPI})$ 

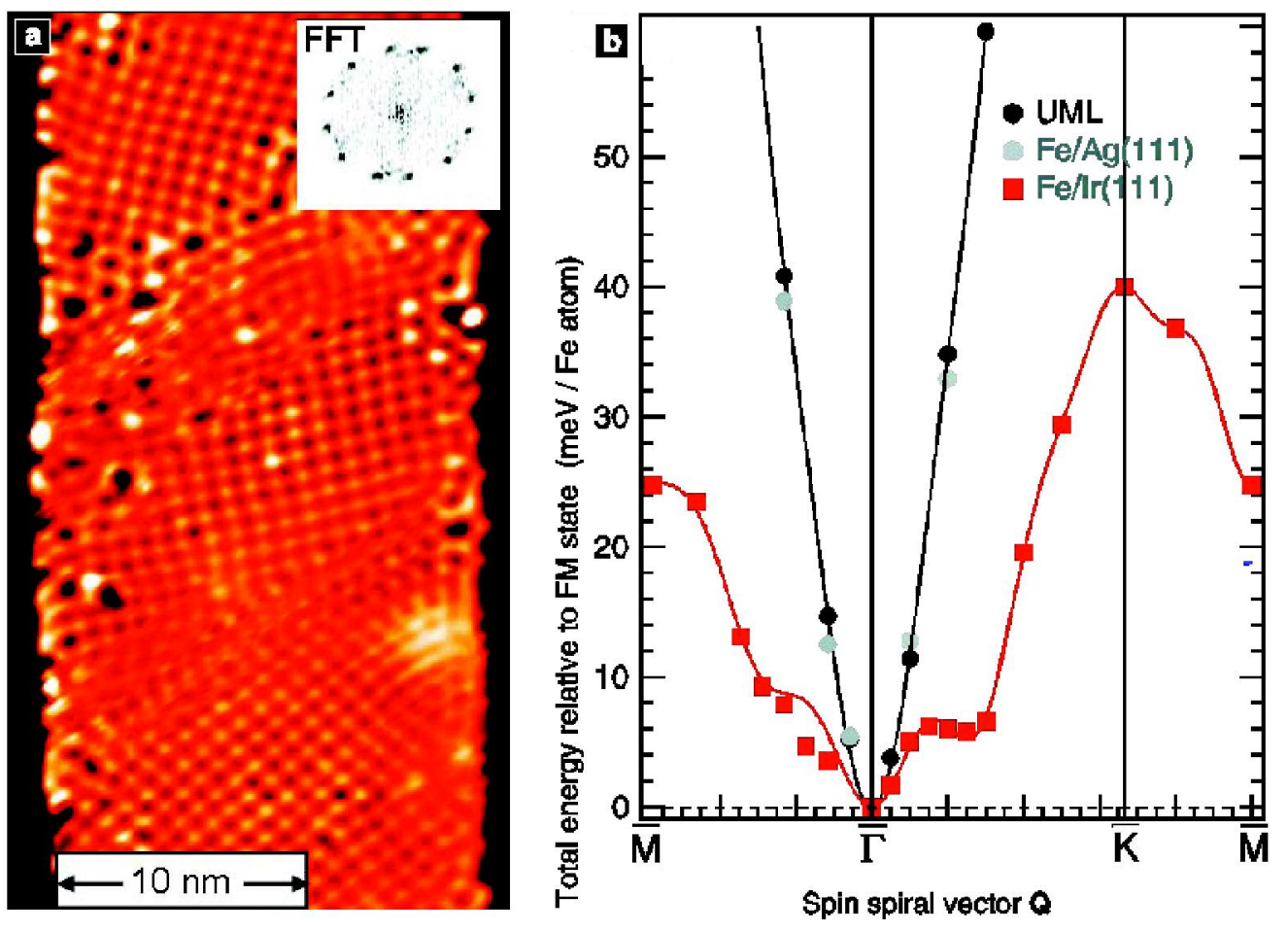

$147 \times 106 \mathrm{~mm}(600 \times 600 \mathrm{DPI})$ 


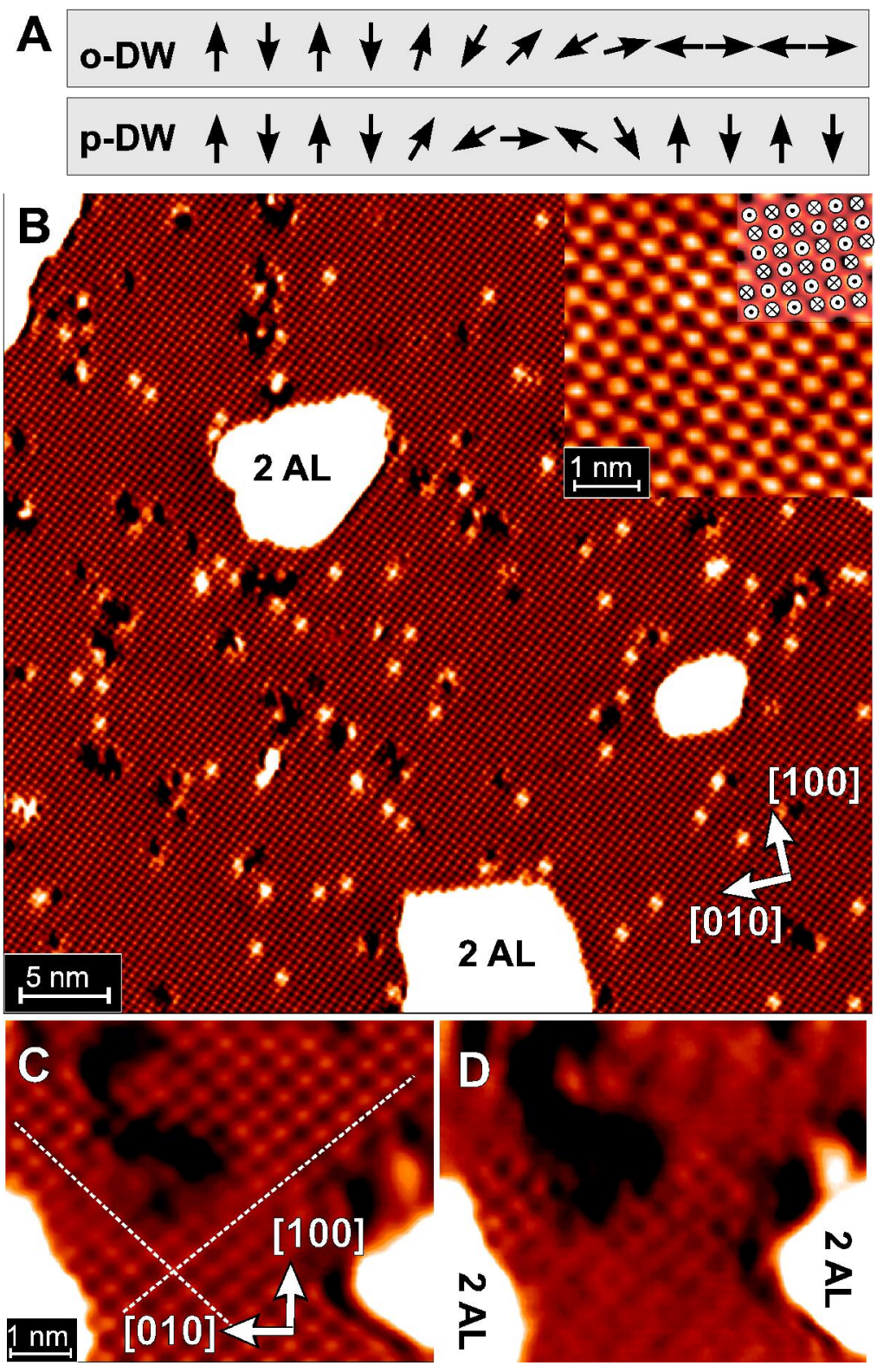

$150 \times 233 \mathrm{~mm}(600 \times 600 \mathrm{DPI})$ 
A

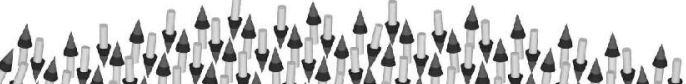
$02 y^{2} y^{2} y^{2} y^{2} y^{2} y^{2} y^{2} y^{2} y^{2} y^{2} y^{4} y^{2} y^{2}$

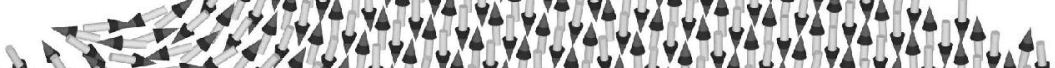

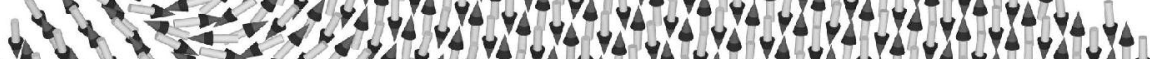

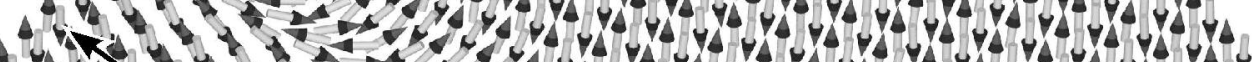
$y^{2} y^{2} y^{2} y^{2}+1+2 y+y^{2} y^{2} y^{2} y^{2} y^{2} y^{2} y^{2} y^{2} y^{2} y^{2} y^{2} y^{2} y^{2}$

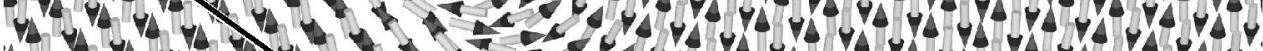

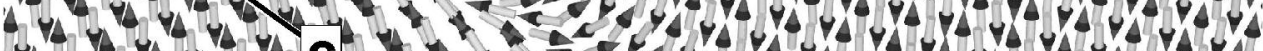

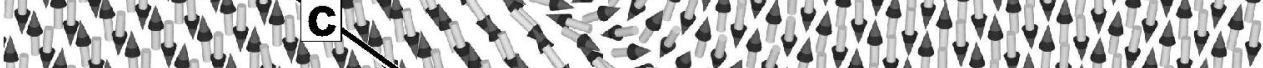

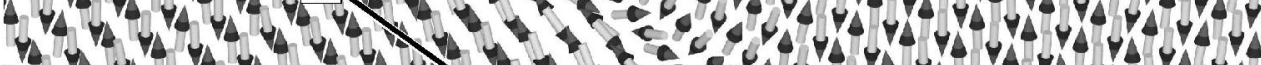

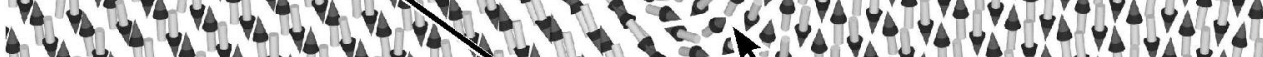

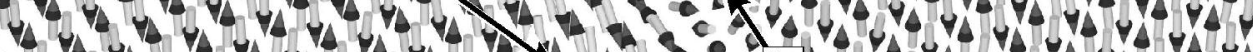

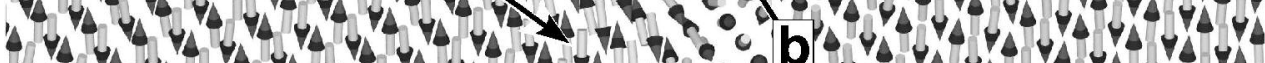

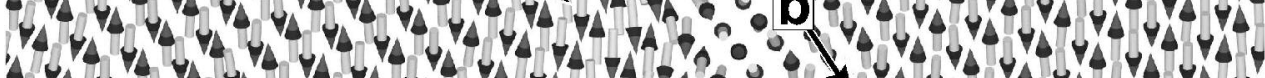

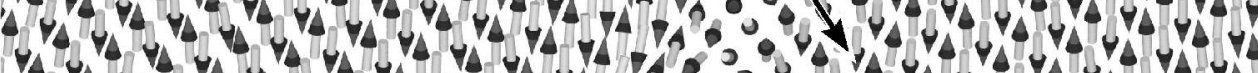

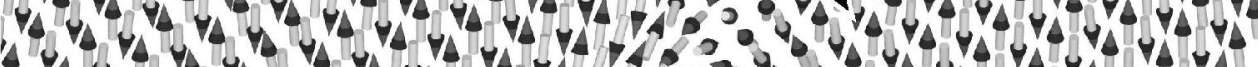

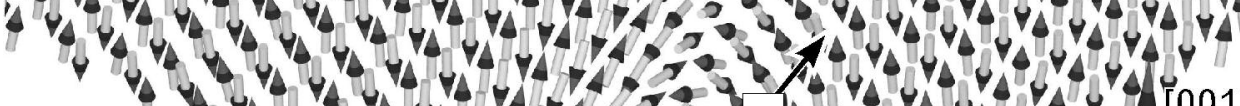

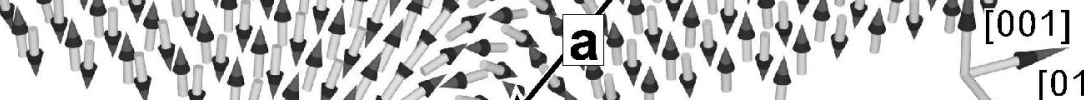

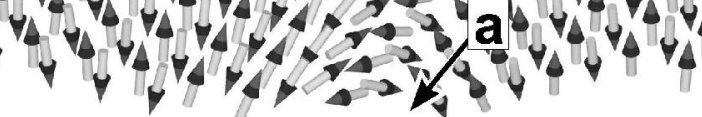

[100]
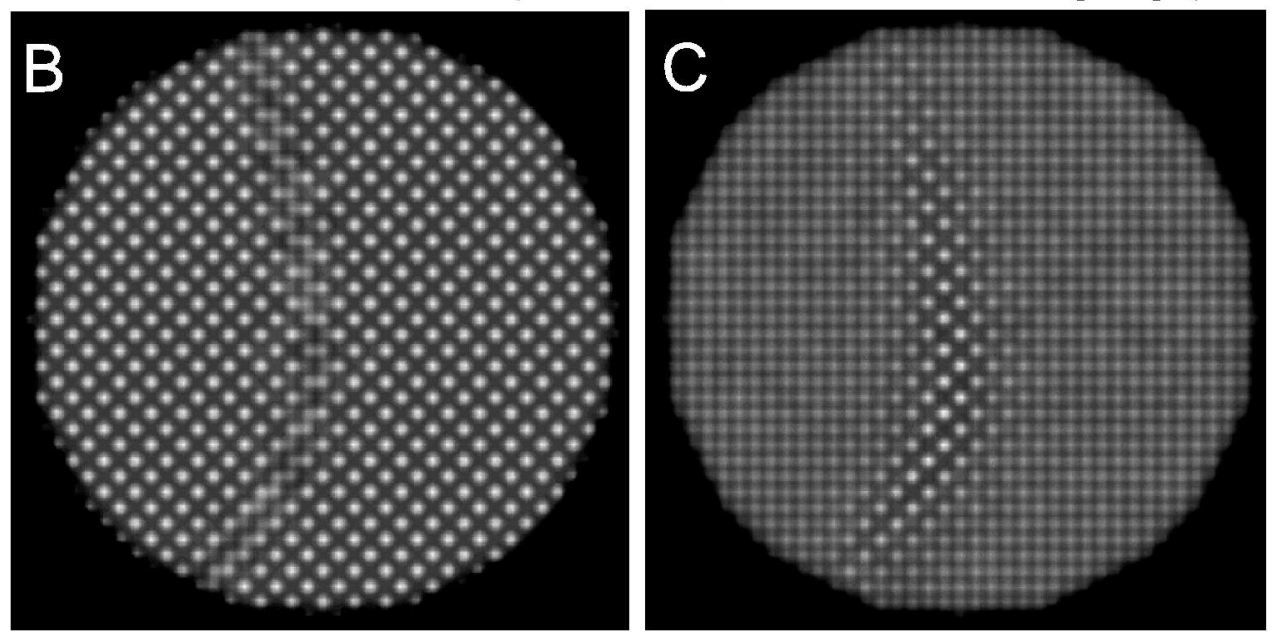

$166 \times 192 \mathrm{~mm}(600 \times 600 \mathrm{DPI})$ 


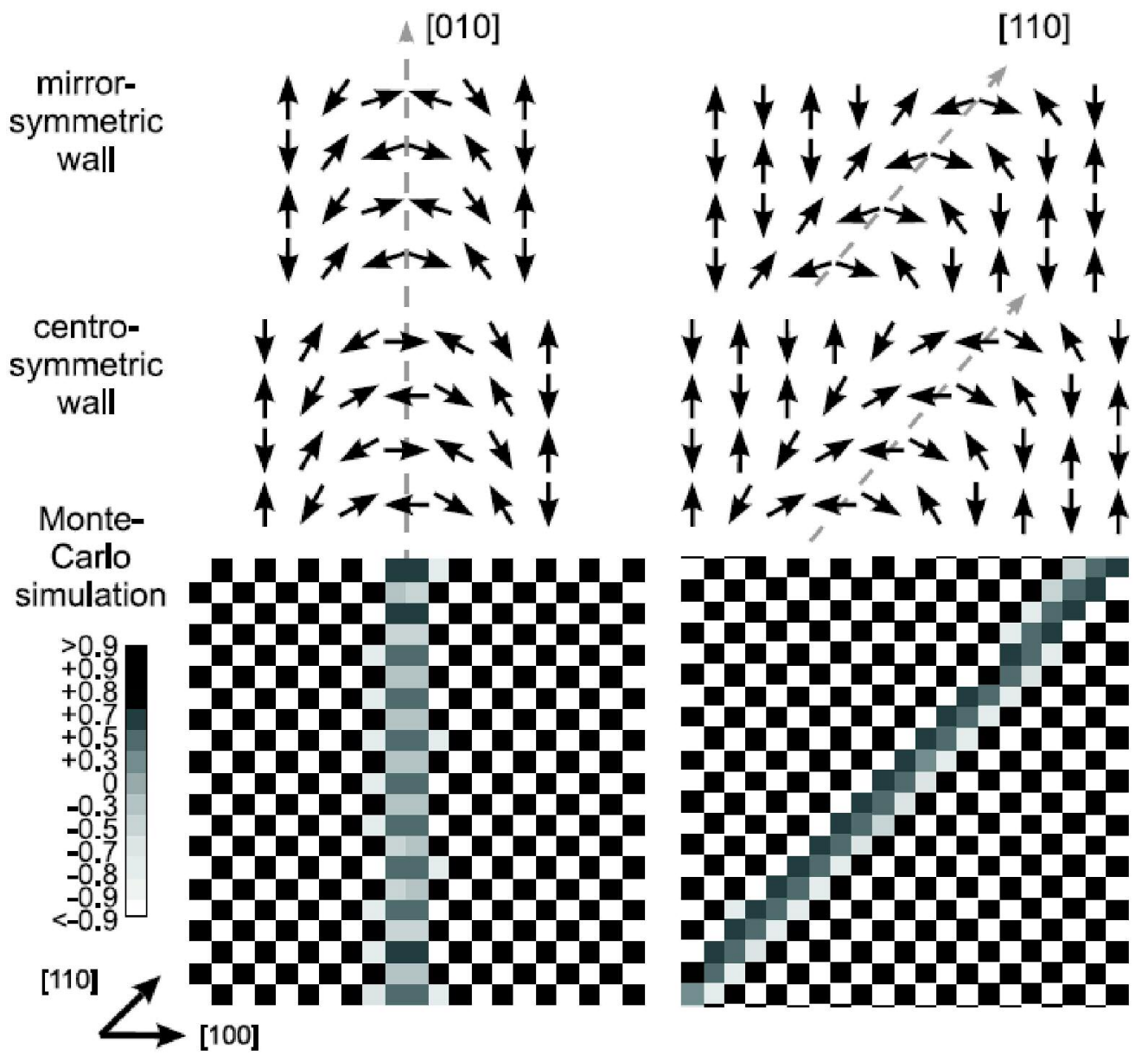

$128 \times 119 \mathrm{~mm}(600 \times 600 \mathrm{DPI})$ 

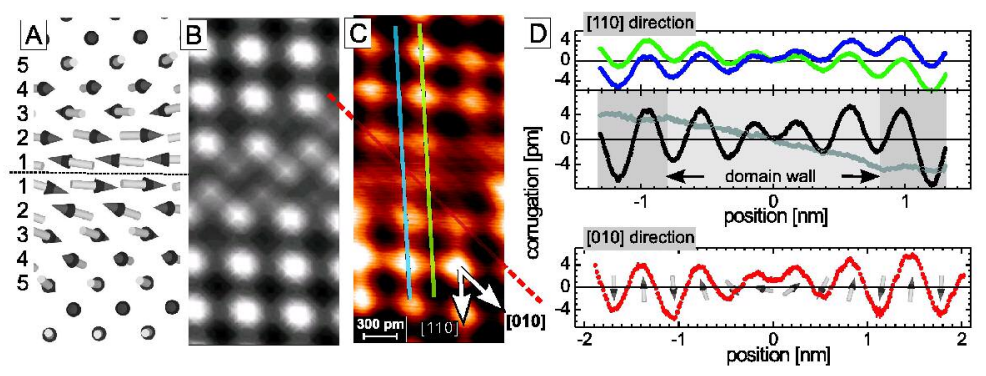

$209 \times 297 \mathrm{~mm}(600 \times 600 \mathrm{DPI})$ 

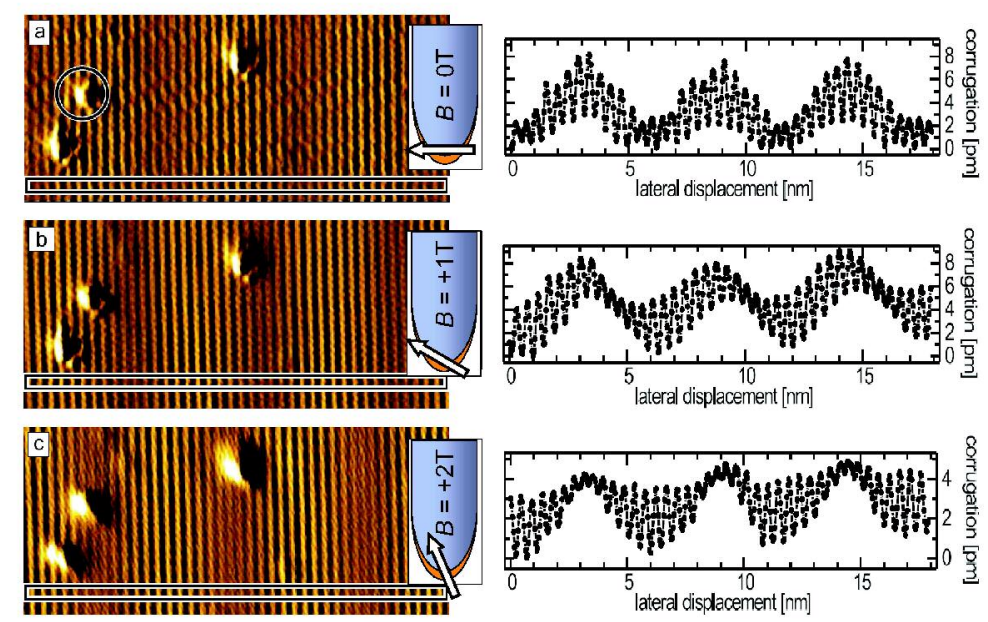

$209 \times 297 m m(600 \times 600$ DPI $)$ 


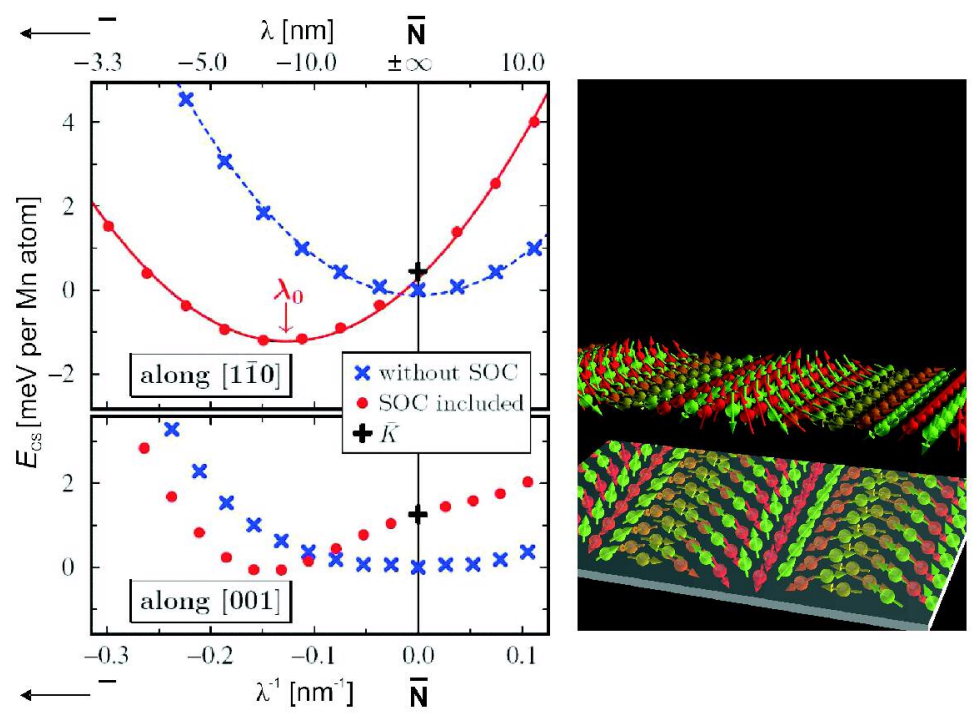

$209 \times 297 \mathrm{~mm}(600 \times 600 \mathrm{DPI})$ 

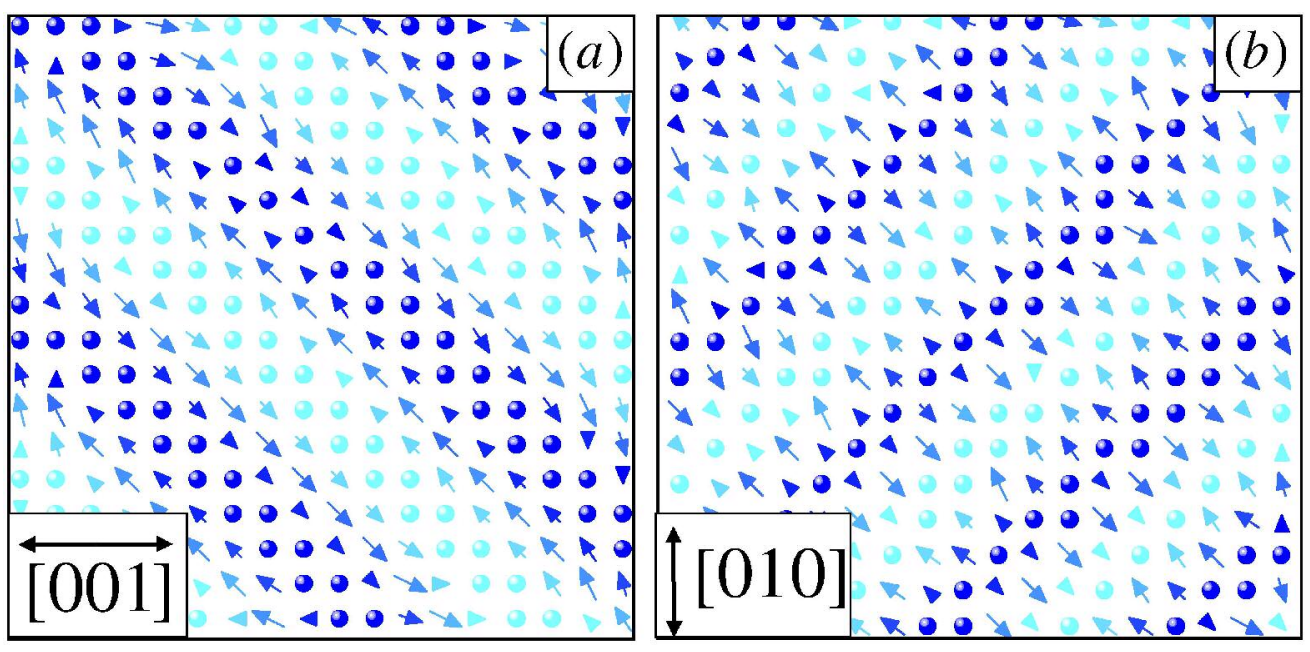

$168 \times 82 \mathrm{~mm}(600 \times 600 \mathrm{DPI})$ 

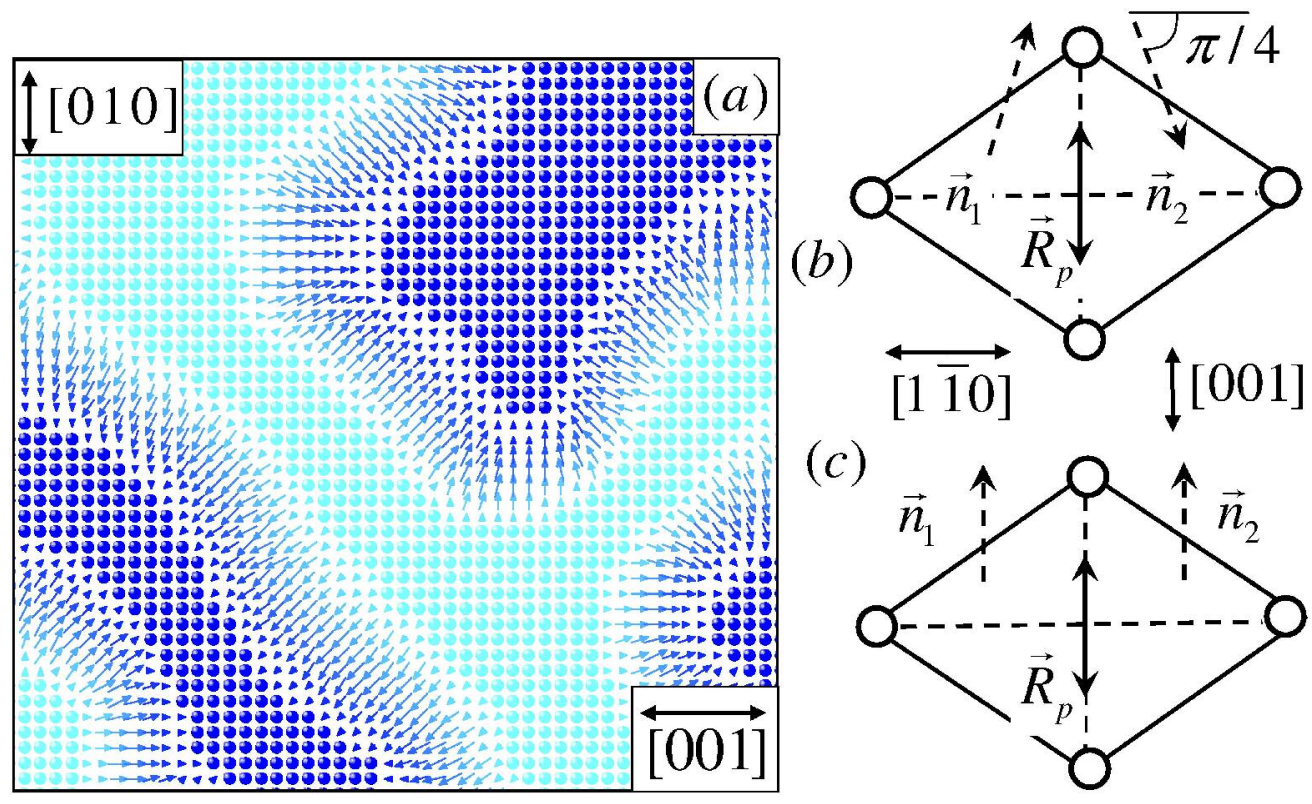

$174 \times 105 \mathrm{~mm}(600 \times 600 \mathrm{DPI})$ 